Real Analysis Exchange

Vol. 28(2), 2002/2003, pp. 287-320

A. Nekvinda, Department of Mathematics, Faculty of Civil Engineering, Czech Technical University, Thákurova 7, 16629 Prague 6, Czech Republic.

e-mail: nekvinda@fsv.cvut.cz

L. Zajíček’ Department of Mathematical Analysis, Charles University, Sokolovská 83, 18600 Prague 8, Czech Republic.

e-mail: zajicek@karlin.mff.cuni.cz

\title{
GÂTEAUX DIFFERENTIABILITY OF LIPSCHITZ FUNCTIONS VIA DIRECTIONAL DERIVATIVES
}

\begin{abstract}
Let $X$ be a separable Banach space, $Y$ a Banach space and $f$ : $X \rightarrow Y$ a Lipschitz function. We show that the set of all Gâteaux non-differentiability points at which $f$ has all one-sided or two-sided directional derivatives can be covered by (special subsets of) Lipschitz surfaces of codimension 1 or codimension 2, respectively. Further results indicate that these results are close to the best possible ones. Our results are new also for Lipschitz functions $\mathbb{R}^{n} \rightarrow \mathbb{R}$; for these functions Gâteaux differentiability is the classical (total) differentiability.
\end{abstract}

\section{Introduction}

In this paper we investigate sets of points $x$ at which a Lipschitz function on a Banach space $X$ is directionally differentiable but $f$ is not Gâteaux differentiable at $x$. These and related sets were treated in a number of articles $([\mathrm{K}]$, [LW], [Fa], [Zh1], [Zh2], [BC] and others; see below for more information). Our results are new also for Lipschitz functions $\mathbb{R}^{n} \rightarrow \mathbb{R}$. For these functions Gâteaux differentiability is the classical (total) differentiability. This simple result is well-known for (different) proofs see e.g. [EG] (p. 83, the proof of the Claim 3) or [NZ1].

\footnotetext{
Key Words: Lipschitz function, directional derivative, Gâteaux differentiability, Lipschitz surface, separable Banach space

Mathematical Reviews subject classification: Primary: 26B05, Secondary: 46G05

Received by the editors April 11, 2002

*The first named author was supported by MSM 210000010

†The second named author was partially supported by grants MSM 113200007 and GAČR 201/00/0767 and GAUK 160/1999
} 
We will use the following terminology.

If $X, Y$ are Banach spaces, $x, u \in X$ and $f$ is a function from $X$ to $Y$, we consider the one-sided directional derivative of $f$ at $x$ in direction $u$

$$
f_{+}^{\prime}(x, u):=\lim _{t \rightarrow 0_{+}} \frac{1}{t}(f(x+t u)-f(x))
$$

and the (two-sided) directional derivative of $f$ at $x$ in direction $u$

$$
f^{\prime}(x, u):=\lim _{t \rightarrow 0} \frac{1}{t}(f(x+t u)-f(x))
$$

If $X, Y$ are Banach spaces, $G \subset X$ is an open set and $f: G \rightarrow Y$ is a Lipschitz function, we will investigate the sets $S_{+}(f), S(f)$ of "singular points" of $f$ defined by

(i) $S_{+}(f)$ is the set of all points $x \in G$ such that $f_{+}^{\prime}(x, u)$ exists for each $u \in X$ but $f$ is not Gâteaux differentiable at $x$.

(ii) $S(f)$ is the set of all points $x \in G$ such that $f^{\prime}(x, u)$ exists for each $u \in X$ but $f$ is not Gâteaux differentiable at $x$.

In particular, we consider the following problems. (They are formulated for real functions, but our results hold for vector functions as well.)

\section{Problem A.}

(i) Characterize the system $\mathcal{S}_{+}(X)$ of all sets which are of the form $S_{+}(f)$ for a Lipschitz real function $f$ on a Banach space $X$.

(ii) Characterize the system $\widetilde{\mathcal{S}}_{+}(X)$ of all sets which are of the form $S_{+}(f)$, where $f$ is a real Lipschitz function which has all one-sided directional derivatives $f_{+}^{\prime}(x, u)$ at all points $x \in X$.

\section{Problem B.}

(i) Characterize the system $\mathcal{S}(X)$ of all sets which are of the form $S(f)$ for a Lipschitz real function $f$ on a Banach space $X$.

(ii) Characterize the system $\widetilde{\mathcal{S}}(X)$ of all sets which are of the form $S(f)$, where $f$ is a real Lipschitz function which has all (two-sided) directional derivatives $f^{\prime}(x, u)$ at all points $x \in X$. 
Note that especially Problem A (ii) is quite natural and therefore we consider Problem A more thoroughly than Problem B. The point is that there exist important types of Lipschitz functions which have all one-sided derivatives at all points. For example, such are quasi-differentiable functions (cf. e.g. $[D V])$ which are used in optimization theory. Further, such functions arise as a supremum (or infimum) of special families of Lipschitz functions. For example $([\mathrm{Z} 2])$, if $X$ is a Banach space with a uniformly Gâteaux differentiable norm and $F \subset X$ is a closed set, then the distance function $x \rightarrow \operatorname{dist}(x, F)$ has all one-sided derivatives at all points $x \in G:=X \backslash F$. Results of [K], [Fa], [Zh1], $[\mathrm{Zh} 2],[\mathrm{FZ}],[\mathrm{FP}],[\mathrm{G}]$ imply that each set from $\mathcal{S}_{+}(X)$ is a first category set in all separable spaces and also in many non-separable spaces. Results of $[\mathrm{BC}]$, [PZ], [Z4], [Z5] even imply that each set from $\mathcal{S}_{+}(X)$ is $\sigma$-porous (in a strong sense) in all separable spaces and in some non-separable spaces. In the case of a separable Banach space we obtain here stronger results.

If $X$ is a separable Banach space, we show that each set of type $S_{+}(f)$ can be covered by countably many Lipschitz surfaces of codimension 1 and each set of type $S(f)$ can be covered by countably many Lipschitz surfaces of codimension 2.

These results were already proved (for real-valued $f$ ) in $[\mathrm{N}]$. Note that the result on $S_{+}(f)$ for distance functions in a separable Banach space with a uniformly Gâteaux differentiable norm is a special case of Theorem 4 from [Z2].

Here we obtain also considerably finer results (Theorem 3.3 and Theorem 3.6) which show that the sets of type $S_{+}(f)$ or $S(f)$ can be covered by countably many "special subsets" of Lipschitz surfaces.

As a consequence we obtain e.g. (cf. Proposition 5.7) that (in each separable space with $\operatorname{dim} X \geq 2$ ) there exists a Lipschitz surface of codimension 1 which cannot be covered by countably many sets of type $S_{+}(f)$. On the other hand, if $A$ is a countable union of "unilaterally smooth" Lipschitz surfaces of codimension 1 in a separable Banach space $X$, then $A \in \widetilde{\mathcal{S}}_{+}(X)$ (cf. Theorem 4.7 for a more precise result). If $\operatorname{dim} X>2$, we do not know whether each set from $\widetilde{\mathcal{S}}_{+}(X)$ can be covered by countably many of "unilaterally smooth" Lipschitz surfaces of codimension 1.

We give a complete solution to Problem $A$ (ii) in the case $X=\mathbb{R}^{2}$. Namely, we obtain (Proposition 5.4) that $\widetilde{\mathcal{S}}_{+}\left(\mathbb{R}^{2}\right)$ is the system of all $F_{\sigma}$ sets which can be covered by countably many "unilaterally smooth Lipschitz curves". (Note that $\widetilde{\mathcal{S}}_{+}(\mathbb{R})=\mathcal{S}_{+}(\mathbb{R})$ is the system of all countable subsets of $\mathbb{R}$, which is an easy and well-known result.)

We also completely solve Problem $B$ (ii) in the case $X=\mathbb{R}^{3}$. Namely (Proposition 5.4) $\widetilde{\mathcal{S}}\left(\mathbb{R}^{3}\right)$ is the system of all $F_{\sigma}$ sets which can be covered 
by countably many "smooth Lipschitz curves". (Our results also show that $\widetilde{\mathcal{S}}\left(\mathbb{R}^{2}\right)=\mathcal{S}\left(\mathbb{R}^{2}\right)$ is the system of all countable sets, which is an easy result.) For a further result concerning Problem B see Theorem 4.10.

We are not able characterize the systems $\mathcal{S}_{+}\left(\mathbb{R}^{2}\right)$ and $\mathcal{S}\left(\mathbb{R}^{3}\right)$, but we give a characterization of $\sigma$-ideals generated by these systems (see Proposition 5.5).

Our results easily imply that in each separable Banach space $X$ there exists a "Lipschitz curve" which cannot be covered by countably many sets from the system $\widetilde{\mathcal{S}}_{+}(X)$ (cf. Proposition 5.8). A further result (see Proposition 5.7) says that, in each separable Banach space with $\operatorname{dim} X \geq 2$, the $\sigma$-ideals generated by the systems $\mathcal{S}_{+}(X)$ and $\widetilde{\mathcal{S}}_{+}(X)$ do not coincide.

Now we describe briefly the structure of the paper. In Section 2 we work with Lipschitz surfaces of finite codimension and their special subsets. This relatively technical but indispensable section is based on a standard application of Bouligand's notion of the contingent (cone) of a set at a point.

In Section 3 we prove that sets of "singular points" of vector valued Lipschitz functions on separable Banach spaces can be covered by special subsets of Lipschitz surfaces (of codimension 1 or 2). Some lemmas (especially Lemma $3.5)$ in this section need relatively delicate arguments.

In Section 4 we present some constructions of Lipschitz functions, which have prescribed "singular points". In the proof of the basic Lemma 4.1 we use the method of B. Kirchheim's proof of Proposition 14 from [PZ]. 5 .

Consequences of the main results are presented in the last section, Section

We finish the introduction with definitions, notation and well-known facts which we will need.

If $f: X \rightarrow Y$ is a mapping and $F \subset X$, then we denote by $f \uparrow_{F}$ the restriction of $f$ to $F$. We use the notation $B(x, r)$ for the open ball with center $x$ and radius $r$.

Definition 1.1. Let $X$ be a Banach space, $A \subset X$ and $x \in X$. Then we denote by $\operatorname{contg}(A, x)$ (the contingent of $A$ at $x$ ) the set of all $0 \neq u \in X$ for which there exist sequences $x \neq x_{i} \in A$ and $\lambda_{i}>0$ such that $\lim \lambda_{i}=0$ and $\lim \frac{1}{\lambda_{i}}\left\|x+\lambda_{i} u-x_{i}\right\|=0$.

For some properties and applications of the contingent see [Sa]. The set $\operatorname{contg}(A, x) \cup\{0\}$ is frequently called the (Bouligand) tangent cone of $A$ at $x$ and denoted by $\operatorname{Tan}(A, x)$ (see [Fe]). Note that clearly $t u \in \operatorname{contg}(A, x)$ whenever $t>0$ and $u \in \operatorname{contg}(A, x)$. It is also easy to see that $\operatorname{contg}(A, x)=$ $\operatorname{contg}(\bar{A}, x)$ and that $a$ is an accumulation point of $A$ whenever $\operatorname{contg}(A, x) \neq$ $\emptyset$. 
Definition 1.2. Let Banach spaces $X, Y$, an open set $G \subset X, x \in G$ and $f: G \rightarrow Y$ be given. Then we put

$$
\operatorname{Lip}(f)=\sup \left\{\frac{\|f(u)-f(v)\|}{\|u-v\|}: u, v \in G, u \neq v\right\}
$$

and

$$
\operatorname{Lip}(f, x)=\limsup _{u \rightarrow x} \frac{\|f(u)-f(x)\|}{\|u-x\|} .
$$

Clearly $\operatorname{Lip}(f, x) \leq \operatorname{Lip}(f)$ and $f$ is $\operatorname{Lipschitz}$ on $G$ iff $\operatorname{Lip}(f)<\infty$.

We will also need the following easy well-known facts.

Lemma 1.3. Let $X, Y, G, f$ be as in Definition 1.2. Then the following assertions hold.

(i) If $f$ is Lipschitz on $G, x \in G$ and $f_{+}^{\prime}(x, u)$ exists for each $u \in X$, then the mapping $u \rightarrow f_{+}^{\prime}(x, u)$ is Lipschitz.

(ii) If $G=X$ and $\operatorname{Lip}(f, x) \leq K$ for each $x \in X$, then $\operatorname{Lip}(f) \leq K$.

For (i) see e.g. [A], p. 164 or [Sp], p. 483. For (ii) see e.g. Lemma 14.4. of $[\mathrm{BL}]$.

\section{$2 \quad$ Lipschitz Surfaces and Their Subsets}

We start with natural definitions of "relative Hadamard" directional derivatives $h_{A,+}^{\prime}(a, v)$ and $h_{A}^{\prime}(a, v)$.

Definition 2.1. Let $Y, W$ be Banach spaces, $A \subset Y, a \in A, v \in Y, d \in W$ and $h: A \rightarrow W$ be a mapping.

(i) We write $h_{A,+}^{\prime}(a, v)=d$ if $v \in \operatorname{contg}(A, a)$ and $\left(1 / \lambda_{i}\right)\left(h\left(x_{i}\right)-h(a)\right) \rightarrow d$ whenever $x_{i} \in A, \lambda_{i}>0, \lambda_{i} \rightarrow 0$ and $\left(1 / \lambda_{i}\right)\left\|a+\lambda_{i} v-x_{i}\right\| \rightarrow 0$.

We say that $h$ is unilaterally smooth at $a$ w.r.t. $A$ if $h_{A,+}^{\prime}(a, v)$ exists for each $v \in \operatorname{contg}(A, a)$.

(ii) We write $h_{A}^{\prime}(a, v)=d$ if $v \in \operatorname{contg}(A, a) \cup(-\operatorname{contg}(A, a))$ and $\left(1 / \lambda_{i}\right)\left(h\left(x_{i}\right)\right.$ $-h(a)) \rightarrow d$ whenever $x_{i} \in A, \lambda_{i} \neq 0, \lambda_{i} \rightarrow 0$ and $\left(1 / \lambda_{i}\right)\left\|a+\lambda_{i} v-x_{i}\right\| \rightarrow$ 0 .

We say that $h$ is bilaterally smooth at $a$ w.r.t. $A$ if $h_{A}^{\prime}(a, v)$ exists for each $v \in \operatorname{contg}(A, a) \cup(-\operatorname{contg}(A, a))$. 
(iii) If $A=Y$, we say that $h$ is unilaterally (or bilaterally) smooth at $a$ if it is unilaterally (or bilaterally) smooth at $a$ w.r.t. $A=Y$.

We say that $h$ is unilaterally (or bilaterally) smooth if it is unilaterally (or bilaterally) smooth at each point of $A=Y$.

Remark 2.2. (i) It is easy to see that $h_{A}^{\prime}(a, v)=d$ iff the following conditions hold:

(a) $v \in \operatorname{contg}(A, a) \cup(-\operatorname{contg}(A, a))$.

(b) If $v \in \operatorname{contg}(A, a)$, then $h_{A,+}^{\prime}(a, v)=d$.

(c) If $v \in-\operatorname{contg}(A, a)$, then $h_{A,+}^{\prime}(a,-v)=-d$.

(ii) Suppose $a \in \operatorname{int} A$. Remark that $h_{A,+}^{\prime}(a, v)$ means in this case the (onesided) Hadamard derivative (cf. [Sp], p. 480). Then clearly

$$
h_{A,+}^{\prime}(a, v)=d \Rightarrow h_{+}^{\prime}(a, v)=d, \quad h_{A}^{\prime}(a, v)=d \Rightarrow h^{\prime}(a, v)=d .
$$

Easy examples show that the opposite implications do not hold in general. On the other hand, it is easy to see that the opposite implications hold if $h$ is Lipschitz (cf. [Sp], Proposition 3.5).

(iii) If $Y=\mathbb{R}$ and $v=1$, then $v \in \operatorname{contg}(A, a)$ iff $a$ is a right accumulation point of $A$. In this case $h_{A,+}^{\prime}(a, v)$ clearly equals to $h_{A,+}^{\prime}(a)$ - the right derivative of $h$ at $a$ w.r.t. $A$.

In the sequel we will need the following natural notion of a Lipschitz surface of codimension $n$ (cf. e.g. [Z7]).

Moreover, we define classes $\mathcal{A}_{n}^{+}, \mathcal{A}_{n}, \mathcal{B}_{n}^{+}, B_{n}$ of "special subsets" of Lipschitz surfaces of codimension $n$, which we will need in the cases $n=1,2$ only.

Definition 2.3. Let $X$ be a Banach space and $0<n \leq \operatorname{dim} X$ be an integer.

(i) We say that $S \subset X$ is a Lipschitz surface of codimension $n$ if there exists a closed linear subspace $Y$ of $X$ of codimension $n$, an $n$-dimensional topological complement $W$ of $Y$ and a Lipschitz mapping $h: Y \rightarrow W$ such that $S=\{y+h(y): y \in Y\}$. Then we say that $S$ is a Lipschitz surface associated with $W$.

If $Y, W, h$ can be chosen in such a way that $h_{+}^{\prime}(y, v)\left(\right.$ or $\left.h^{\prime}(y, v)\right)$ exists for each $y, v \in Y$, we say that $S$ is a unilaterally smooth (or a bilaterally smooth) Lipschitz surface associated with $W$.

If $\operatorname{dim}(Y)=1$, we will speak about Lipschitz curves, unilaterally smooth Lipschitz curves and smooth Lipschitz curves. 
(ii) Let $Z \subset X$ and there exist $Y, W, h, S$ as above such that $Z \subset S$. Let $\pi_{Y}$ be the projection of $X$ on $Y$ parallel to $W$. We say that

(a) $Z \in \mathcal{A}_{n}^{+}$if $h$ is unilaterally smooth w.r.t. $\pi_{Y}(Z)$ at each point $a \in \pi_{Y}(Z)$.

(b) $Z \in \mathcal{A}_{n}$ if $h$ is bilaterally smooth w.r.t. $\pi_{Y}(Z)$ at each point $a \in$ $\pi_{Y}(Z)$.

(c) $Z \in \mathcal{B}_{n}^{+}$if $h$ has all one-sided directional derivatives $h_{+}^{\prime}(a, y), y \in Y$, at each point $a \in \pi_{Y}(Z)$.

(d) $Z \in \mathcal{B}_{n}$ if $h$ has all (two-sided) directional derivatives $h^{\prime}(a, y), y \in Y$, at each point $a \in \pi_{Y}(Z)$.

Remark 2.4. (i) We admit also the trivial case $n=\operatorname{dim} X$. In this case Lipschitz surfaces are singletons and $\mathcal{A}_{n}^{+}=\mathcal{A}_{n}=\mathcal{B}_{n}^{+}=\mathcal{B}_{n}$ is the system containing all singletons and $\emptyset$.

(ii) Using Remark 2.2 (ii), we immediately obtain

$$
\mathcal{B}_{n}^{+} \subset \mathcal{A}_{n}^{+}, \mathcal{B}_{n} \subset \mathcal{A}_{n}, \mathcal{A}_{n} \subset \mathcal{A}_{n}^{+}, \mathcal{B}_{n} \subset \mathcal{B}_{n}^{+} .
$$

(iii) A Lipschitz surface of codimension $n$ in $X$ is clearly closed.

Let $S, Y, W$ be as in Definition 2.3 (i) and $\pi_{Y}$ be the projection of $X$ on $Y$ parallel to $W$. It is easy to see that $\pi_{Y}\left\lceil_{S}: S \rightarrow Y\right.$ is a bilipschitz bijection, in particular $\pi_{Y}(A)$ is closed iff $A \subset S$ is closed.

(iv) We will also write $\mathcal{A}_{n}^{+}(X), \mathcal{A}_{n}(X), \ldots$ instead of $\mathcal{A}_{n}^{+}, \mathcal{A}_{n}, \ldots$ when it is not clear in which space $X$ we are working.

Now we will show how extension theorems give some non-trivial relations among defined classes in the cases $\operatorname{dim} X=2$ and $\operatorname{dim} X=3$. We will use the terminology of Definition 2.1; cf. also Remark 2.2, (iii).

The first extension result is essentially contained in $[\mathrm{J}]$.

Lemma 2.5. Let $A \subset \mathbb{R}$ and $f: A \rightarrow \mathbb{R}$ be a Lipschitz function which is unilaterally smooth w.r.t $A$ at each point of $A$. Then there exists a Lipschitz function $F: \mathbb{R} \rightarrow \mathbb{R}$ such that $F \uparrow_{A}=f, \operatorname{Lip}(F)=\operatorname{Lip}(f)$ and $F_{+}^{\prime}(x), F_{-}^{\prime}(x)$ exist for all $x \in A \cup(\mathbb{R} \backslash \bar{A})$.

Proof. Clearly there exists a unique function $f^{*}$ defined on $\bar{A}$ such that $\operatorname{Lip}\left(f^{*}\right)=\operatorname{Lip}(f)$ and $\left.f^{*}\right|_{A}=f$. It is easy to see that $f^{*}$ is unilaterally smooth w.r.t. $\bar{A}$ at each point of $A$. Let $\left\{I_{s}=\left(a_{s}, b_{s}\right): s \in S\right\}$ be the system of all components of $\mathbb{R} \backslash \bar{A}$. Let $F$ be the (clearly unique) extension of $f^{*}$ with 
the following properties: If $I_{s}$ is bounded, then $F$ is linear on $\overline{I_{s}}$. If $I_{s}$ is unbounded, then $F$ is constant on $\overline{I_{s}}$. It is easy to verify $\operatorname{Lip}(F)=\operatorname{Lip}(f)$. To prove that $F_{+}^{\prime}(x)$ and $F_{-}^{\prime}(x)$ exist for all $x \in A$ we repeat the argument of Jarník [J]: It is sufficient to observe that if $y$ belongs to a bounded $\left(a_{s}, b_{s}\right)$ with $x \neq a_{s}, x \neq b_{s}$, then $\frac{F(y)-F(x)}{y-x}$ is between $\frac{f^{*}\left(a_{s}\right)-f(x)}{a_{s}-x}$ and $\frac{f^{*}\left(b_{s}\right)-f(x)}{b_{s}-x}$.

The second extension theorem we need follows immediately from $[\mathrm{M}]$. Note that we cannot use results from $[\mathrm{J}]$, since Jarník in $[\mathrm{J}]$ extends functions from perfect sets only and does not discuss the "Lipschitz case".

Lemma 2.6. Let $C \subset \mathbb{R}$ be closed and $h: C \rightarrow \mathbb{R}$ be a Lipschitz function which is bilaterally smooth w.r.t $C$ at each point $a \in C$. Then there exists a Lipschitz function $g: \mathbb{R} \rightarrow \mathbb{R}$ such that $g\left\lceil_{C}=h\right.$ and $g^{\prime}(x)$ exists for all $x \in \mathbb{R}$.

Proof. Set $S:=C, F:=h, A:=-\operatorname{Lip}(h)-1$ and $B:=\operatorname{Lip}(h)+1$. It is easy to see that then we can use Theorem 8 of $[\mathrm{M}]$ to obtain a function $g$ with the desired properties.

We will also need the following extension result, which can be proved by modifications of constructions from $[\mathrm{J}]$ or $[\mathrm{M}]$. It is a special case of the main result of $[\mathrm{NZ2}]$ where we use the ideas of $[\mathrm{J}]$.

Lemma 2.7. Let $A \subset \mathbb{R}$ be an arbitrary set and $h: A \rightarrow \mathbb{R}$ be a Lipschitz function which is bilaterally smooth w.r.t. A at each point $a \in A$. Then there exists a Lipschitz function $g: \mathbb{R} \rightarrow \mathbb{R}$ such that $g\left\lceil_{A}=h\right.$ and $g^{\prime}(x)$ exists for all $x \in A$.

Lemma 2.8. Let $X=\mathbb{R}^{2}$. Then $\mathcal{A}_{1}^{+}(X)=\mathcal{B}_{1}^{+}(X)$. Moreover, each closed set from $\mathcal{A}_{1}^{+}(X)$ is contained in an unilaterally smooth Lipschitz curve.

Proof. Let $Z \in \mathcal{A}_{1}^{+}(X)$. By Definition 2.3 there exist two one-dimensional subspaces $Y, W$ of $X$ with $X=Y \oplus W$ and a Lipschitz function $g: Y \rightarrow W$ such that $Z \subset S:=\{y+g(y): y \in Y\}$ and $g$ is unilaterally smooth w.r.t. $P(Z)$ at each point $a \in P(Z)$, where $P$ is the projection of $X$ on $Y$ parallel to $W$. Since both $Y$ and $W$ are (linearly) isometric to $\mathbb{R}$, Lemma 2.5 implies that there exists a Lipschitz function $g^{*}: Y \rightarrow W$ such that $g \uparrow_{P(Z)}=g^{*} \uparrow_{P(Z)}$ and $g^{*}$ is unilaterally smooth at all points of $P(Z) \cup(Y \backslash \overline{P(Z)})$. If $Z$ is closed, then $P(Z)$ is closed (cf. Remark 2.4 (iii)) and therefore $g^{*}$ is unilaterally smooth. Thus the assertion of the lemma follows immediately.

Lemma 2.9. Let $X=\mathbb{R}^{3}$. Then $\mathcal{A}_{2}(X)=\mathcal{B}_{2}(X)$. Moreover, if $Z \in \mathcal{A}_{2}(X)$ is closed, then $Z$ is contained in a smooth Lipschitz curve. 
Proof. Let $Z \in \mathcal{A}_{2}(X)$. By Definition 2.3 there exist a closed one-dimensional subspace $Y$ of $X$, a 2-dimensional topological complement $W$ to $Y$ and a Lipschitz function $g: Y \rightarrow W$ such that $Z \subset S:=\{y+g(y): y \in Y\}$ and $g$ is bilaterally smooth w.r.t. $P(Z)$ at each point $a \in P(Z)$, where $P$ is the projection of $X$ on $Y$ parallel to $W$. Let $W=\operatorname{span}\left\{w_{1}, w_{2}\right\}$ and $g_{1}, g_{2}: Y \rightarrow \mathbb{R}$ be the functions for which $g(y)=g_{1}(y) w_{1}+g_{2}(y) w_{2}$. Since $Y$ is (linearly) isometric to $\mathbb{R}$ and $g_{1}, g_{2}$ are clearly bilaterally smooth w.r.t. $P(Z)$ at each point $a \in P(Z)$, by Lemma 2.7 there are Lipschitz extensions $g_{1}^{*}: Y \rightarrow \mathbb{R}, g_{2}^{*}: Y \rightarrow \mathbb{R}$ of $g_{1} \uparrow_{P(Z)}, g_{2} \uparrow_{P(Z)}$ such that $\left(g_{1}^{*}\right)^{\prime}(x),\left(g_{2}^{*}\right)^{\prime}(x)$ exist for all $x \in P(Z)$. Moreover, if $Z$ is closed, Lemma 2.6 gives that we can even obtain that $\left(g_{1}^{*}\right)^{\prime}(x),\left(g_{2}^{*}\right)^{\prime}(x)$ exist for all $x \in Y$. If we now put $g^{*}(y):=g_{1}^{*}(y) w_{1}+g_{2}^{*}(y) w_{2}$ and consider the Lipschitz curve $S^{*}:=\left\{y+g^{*}(y): y \in Y\right\}$, the inclusion $Z \subset S^{*}$ proves the lemma.

We do not know whether each closed set from $\mathcal{A}_{1}^{+}$belongs to $\mathcal{B}_{1}^{+}$, if $\operatorname{dim} X>$ 2. We also do not know whether each closed set from $\mathcal{A}_{2}$ belongs to $\mathcal{B}_{2}$, if $\operatorname{dim} X>3$. The following lemma is well-known in Euclidean spaces (cf. [Sa]) and was proved and used in [Z1] in the case $\operatorname{dim} W=1$.

Lemma 2.10. Let $X$ be a Banach space and $W$ a finite-dimensional subspace of $X$ with $\operatorname{dim} W \geq 1$. Let $A \subset X$ and let $\operatorname{contg}(A, a) \cap W=\emptyset$ for each $a \in A$. Then $A$ can be covered by countably many Lipschitz surfaces associated with $W$.

Proof. Let $V$ be a topological complement of $W$. Let $P_{w}$ and $P_{v}$ be projections of $X$ onto $W$ and $V$ parallel to $W$ and $V$, respectively. Then for each $x \in A$ there exists $n \in \mathbb{N}$ such that

$$
\left(y \in A,\left\|P_{w}(y-x)\right\| \leq \frac{1}{n}\right) \Rightarrow\left\|P_{v}(y-x)\right\| \geq \frac{1}{n}\left\|P_{w}(y-x)\right\| .
$$

Assume for the moment the contrary. Then there exist $x \in A$ and a sequence $y_{n} \in A$ such that

$$
\left\|P_{w}\left(y_{n}-x\right)\right\| \leq \frac{1}{n} \text { and }\left\|P_{v}\left(y_{n}-x\right)\right\|<\frac{1}{n}\left\|P_{w}\left(y_{n}-x\right)\right\| .
$$

Put $z_{n}:=y_{n}-x$. By $(2.2)$ we have $P_{w}\left(z_{n}\right) \neq 0$ and $P_{w}\left(z_{n}\right) \rightarrow 0$. Since the unit sphere of $W$ is compact, we can find $u \in W,\|u\|=1$, and a subsequence $\left\{P_{w}\left(z_{n_{k}}\right)\right\}$ of $\left\{P_{w}\left(z_{n}\right)\right\}$ such that $\left\|P_{w}\left(z_{n_{k}}\right)\right\|^{-1} P_{w}\left(z_{n_{k}}\right) \rightarrow u$. Putting $t_{k}:=$ $\left.\| P_{w} z_{n_{k}}\right) \|$, we obtain

$$
t_{k} \rightarrow 0 \text { and } \frac{1}{t_{k}}\left\|t_{k} u-P_{w}\left(z_{n_{k}}\right)\right\| \rightarrow 0
$$


Since $z_{n_{k}}=P_{w}\left(z_{n_{k}}\right)+P_{v}\left(z_{n_{k}}\right)$ and $t_{k}=\left\|P_{w}\left(z_{n_{k}}\right)\right\|,(2.2)$ and (2.3) give

$$
\frac{1}{t_{k}}\left\|t_{k} u-z_{n_{k}}\right\| \leq \frac{1}{t_{k}}\left\|t_{k} u-P_{w}\left(z_{n_{k}}\right)\right\|+\frac{1}{t_{k}}\left\|P_{v}\left(z_{n_{k}}\right)\right\| \rightarrow 0 .
$$

Thus $u \in \operatorname{contg}(M, x)$ which is a contradiction.

Let $A_{n}$ be the set of all points $x \in A$, for which (2.1) holds. We have proved $A=\bigcup_{n=1}^{\infty} A_{n}$. Fix $n \in \mathbb{N}$. Since $\operatorname{dim} W<\infty$ we can clearly write $W=\bigcup_{m=1}^{\infty} B_{m}$ where $\operatorname{diam} B_{m}<\frac{1}{n}$ for each $m \in \mathbb{N}$. Putting

$$
A_{n, m}=\left\{x \in A_{n}: P_{w}(x) \in B_{m}\right\}
$$

we obtain $A=\bigcup_{n, m=1}^{\infty} A_{n, m}$. If $x, y \in A_{n, m}$, then we have $\left\|P_{w}(y-x)\right\|<\frac{1}{n}$ and consequently, by the definition of $A_{n},\left\|P_{w}(y-x)\right\| \leq n\left\|P_{v}(y-x)\right\|$. Therefore the set $\left\{\left(P_{v}(x), P_{w}(x)\right): x \in A_{n, m}\right\}$ is a graph of a Lipschitz function $f_{n, m}: P_{v}\left(A_{n, m}\right) \rightarrow W$. There exists a Lipschitz function $\widetilde{f}_{n, m}: V \rightarrow W$ which extends $f_{n, m}$; it easily follows from the well-known results (see for instance $[\mathrm{Sn}]$ ) on extensions of real-valued functions. Thus $A_{n, m}$ is a subset of a Lipschitz surface associated with $W$, which finishes the proof.

Lemma 2.11. Let $X$ be a Banach space, $Y$ be a closed linear subspace of $X$ of codimension $n, W$ be a topological complement of $Y$ in $X$ and $h: Y \rightarrow W$ be a Lipschitz mapping. Let $S=\{y+h(y): y \in Y\}$ and $Z \subset S$ be given. Then the following statements hold.

(i) If $(\operatorname{contg}(Z, z)-\operatorname{contg}(Z, z)) \cap W \subset\{0\}$ for each $z \in Z$, then $Z \in \mathcal{A}_{n}^{+}$.

(ii) If $(\operatorname{contg}(Z, z)-\operatorname{contg}(Z, z)) \cap W \subset\{0\}$ and $(\operatorname{contg}(Z, z)+\operatorname{contg}(Z, z)) \cap$ $W \subset\{0\}$ for each $z \in Z$, then $Z \in \mathcal{A}_{n}$.

Proof. Denote by $\pi_{Y}$ the projection of $X$ on $Y$ parallel to $W$ and put $A:=$ $\pi_{Y}(Z)$. To prove (i), suppose that $Z \notin \mathcal{A}_{n}^{+}$. Then there exist $a \in A, a=$ $\pi_{Y}(z)$, and $v \in Y$ such that $v \in \operatorname{contg}(A, a)$ and $h_{A,+}^{\prime}(a, v)$ does not exist. Consequently there exist sequences $x_{i} \in A, \lambda_{i}>0$ such that

$$
\lambda_{i} \rightarrow 0 \text { and }\left(1 / \lambda_{i}\right)\left\|a+\lambda_{i} v-x_{i}\right\| \rightarrow 0
$$

but $\lim _{i \rightarrow \infty}\left(1 / \lambda_{i}\right)\left(h\left(x_{i}\right)-h(a)\right)$ does not exist. Since

$$
q_{i}:=\left\|\frac{h\left(x_{i}\right)-h(a)}{\lambda_{i}}\right\| \leq \operatorname{Lip}(h) \frac{\left\|x_{i}-a\right\|}{\lambda_{i}} \leq \operatorname{Lip}(h) \frac{\left\|\lambda_{i} v\right\|+\left\|a+\lambda_{i} v-x_{i}\right\|}{\lambda_{i}},
$$


(2.4) gives that the sequence $\left(q_{i}\right)$ is bounded. Since $W$ is finite-dimensional, we can find increasing sequences of natural numbers $i(k), i^{*}(k)$ and elements $d \neq d^{*}$ of $W$ such that

$$
\lim _{k \rightarrow \infty} \frac{h\left(x_{i(k)}\right)-h(a)}{\lambda_{i(k)}}=d, \quad \lim _{k \rightarrow \infty} \frac{h\left(x_{i^{*}(k)}\right)-h(a)}{\lambda_{i^{*}(k)}}=d^{*} .
$$

By the definition of $A$ we have

$$
z_{k}:=x_{i(k)}+h\left(x_{i(k)}\right) \in Z, z_{k}^{*}:=x_{i^{*}(k)}+h\left(x_{i^{*}(k)}\right) \in Z .
$$

Since clearly $z_{k} \rightarrow z$ and

$$
\begin{aligned}
\frac{1}{\lambda_{i(k)}}\left\|z+\lambda_{i(k)}(v+d)-z_{k}\right\| \leq & \frac{1}{\lambda_{i(k)}}\left\|a-x_{i(k)}-\lambda_{i(k)} v\right\| \\
& +\frac{1}{\lambda_{i(k)}}\left\|h(a)-h\left(x_{i(k)}\right)-\lambda_{i(k)} d\right\| \rightarrow 0,
\end{aligned}
$$

we obtain $v+d \in \operatorname{contg}(Z, z)$. Quite analogously we obtain $v+d^{*} \in$ $\operatorname{contg}(Z, z)$. Consequently $0 \neq d-d^{*} \in(\operatorname{contg}(Z, z)-\operatorname{contg}(Z, z)) \cap W$ which is a contradiction.

To prove (ii), we suppose that $Z \notin \mathcal{A}_{n}$ and obtain $a \in A, a=\pi_{Y}(z)$, and $v \in Y$ such that $v \in \operatorname{contg}(A, a) \cup(-\operatorname{contg}(A, a))$ and $h_{A}^{\prime}(a, v)$ does not exist. Further we proceed quite similarly to the proof of (i). We obtain $x_{i} \in A$ and $\lambda_{i}$ as above, with the only difference that now $\lambda_{i} \neq 0$ (instead of $\lambda_{i}>0$ ). Passing to the subsequences $\lambda_{i(k)}, \lambda_{i^{*}(k)}$, we can clearly suppose that their signs are constant, but possibly different. If $\operatorname{sign}\left(\lambda_{i(k)}\right)=\operatorname{sign}\left(\lambda_{i^{*}(k)}\right)=1$, we obtain a contradiction in the same way as above. If $\operatorname{sign}\left(\lambda_{i(k)}\right)=\operatorname{sign}\left(\lambda_{i^{*}(k)}\right)=-1$, then we easily obtain $-v-d \in \operatorname{contg}(Z, z),-v-d^{*} \in \operatorname{contg}(Z, z)$,

$$
0 \neq d-d^{*} \in(\operatorname{contg}(Z, z)-\operatorname{contg}(Z, z)) \cap W,
$$

which is a contradiction. If $\operatorname{sign}\left(\lambda_{i(k)}\right)=1$ and $\operatorname{sign}\left(\lambda_{i^{*}(k)}\right)=-1$, then $v+d \in \operatorname{contg}(Z, z),-v-d^{*} \in \operatorname{contg}(Z, z)$,

$$
0 \neq d-d^{*} \in(\operatorname{contg}(Z, z)+\operatorname{contg}(Z, z)) \cap W,
$$

which is a contradiction.

Remark 2.12. It is not difficult to prove that the opposite implications in (i) and (ii) also hold. 


\section{$3 \quad$ Properties of Sets of Singular Points}

In the following we suppose that $X, Y$ are Banach spaces, $G \subset X$ is an open set and $f: G \rightarrow Y$ is a Lipschitz mapping.

Lemma 3.1. Let $A \subset G, x_{0} \in G, u, c \in X, U \in Y, \delta>0, c \in \operatorname{contg}\left(A, x_{0}\right)$ and $\varepsilon>0$ be given. Then the following assertions hold.

(i) If the inequality $\left\|\frac{f(x+t u)-f(x)}{t}-U\right\| \leq \varepsilon$ holds for each point $x \in A$ and $0<t<\delta$, then

$$
\left\|f_{+}^{\prime}\left(x_{0}, c+\lambda u\right)-f_{+}^{\prime}\left(x_{0}, c\right)-\lambda U\right\| \leq \lambda \varepsilon
$$

whenever $\lambda>0$ and the one-sided directional derivatives $f_{+}^{\prime}\left(x_{0}, c\right), f_{+}^{\prime}\left(x_{0}, c+\right.$ $\lambda u)$ exist.

(ii) If the inequality $\left\|\frac{f(x+t u)-f(x)}{t}-U\right\|<\varepsilon$ holds for each $x \in A$ and $0<$ $|t|<\delta$, then

$$
\left\|f_{+}^{\prime}\left(x_{0}, c+\lambda u\right)-f_{+}^{\prime}\left(x_{0}, c\right)-\lambda U\right\| \leq|\lambda| \varepsilon
$$

whenever $\lambda \in \mathbb{R}$ and the one-sided directional derivatives $f_{+}^{\prime}\left(x_{0}, c\right), f_{+}^{\prime}\left(x_{0}, c+\right.$ $\lambda u)$ exist.

Proof. By the definition of the contingent, we can choose a sequence of numbers $t_{n}>0, t_{n} \rightarrow 0$ and a sequence of points $x_{n} \in A$ such that

$$
d_{n}:=\left\|x_{n}-\left(x_{0}+t_{n} c\right)\right\|=o\left(t_{n}\right), n \rightarrow \infty .
$$

Suppose that the assumptions of (i) are satisfied and a $\lambda>0$ is given. We have

$$
\begin{aligned}
V_{n}:= & \left\|\frac{f\left(x_{0}+t_{n}(c+\lambda u)\right)-f\left(x_{0}\right)}{t_{n}}-\frac{f\left(x_{0}+t_{n} c\right)-f\left(x_{0}\right)}{t_{n}}-\lambda U\right\| \\
\leq & \left\|\lambda\left(\frac{f\left(x_{n}+t_{n} \lambda u\right)-f\left(x_{n}\right)}{\lambda t_{n}}-U\right)\right\| \\
& +\left\|\frac{f\left(x_{0}+t_{n}(c+\lambda u)\right)-f\left(x_{n}+t_{n} \lambda u\right)}{t_{n}}\right\| \\
& +\left\|\frac{f\left(x_{0}+t_{n} c\right)-f\left(x_{n}\right)}{t_{n}}\right\|:=\alpha_{n}+\beta_{n}+\gamma_{n} .
\end{aligned}
$$

By our assumptions $\alpha_{n} \leq \lambda \varepsilon$ for all sufficiently large $n$. Since clearly $\beta_{n} \leq$ $\operatorname{Lip}(f) \frac{d_{n}}{t_{n}}$ and $\gamma_{n} \leq \operatorname{Lip}(f) \frac{d_{n}}{t_{n}}$, we obtain

$$
\begin{aligned}
\left\|f_{+}^{\prime}\left(x_{0}, c+\lambda u\right)-f_{+}^{\prime}\left(x_{0}, c\right)-\lambda U\right\| & =\lim _{n \rightarrow \infty} V_{n} \\
& \leq \lambda \varepsilon+0+0=\lambda \varepsilon
\end{aligned}
$$


which proves (i). To prove (ii), consider a negative $\lambda$. Put $\widetilde{\lambda}:=-\lambda, \widetilde{u}:=$ $-u, \widetilde{U}:=-U$. Since $\left\|\frac{f(x+t \widetilde{u})-f(x)}{t}-\widetilde{U}\right\|<\varepsilon$ for $0<t<\delta$, by (i) we obtain

$$
\begin{aligned}
\left\|f_{+}^{\prime}\left(x_{0}, c+\lambda u\right)-f_{+}^{\prime}\left(x_{0}, c\right)-\lambda U\right\| & =\left\|f_{+}^{\prime}\left(x_{0}, c+\widetilde{\lambda} \widetilde{u}\right)-f_{+}^{\prime}\left(x_{0}, c\right)-\widetilde{\lambda} \widetilde{U}\right\| \\
& \leq \widetilde{\lambda} \varepsilon=|\lambda| \varepsilon .
\end{aligned}
$$

Lemma 3.2. Let $M \subset G, x_{0} \in G, u, v, w, s_{1}, s_{2} \in X, \varepsilon, \delta>0, U, V, W \in Y$ be given such that $w=u+v,\|W-(U+V)\|>3 \varepsilon, s_{1}, s_{2} \in \operatorname{contg}\left(M, x_{0}\right)$ and $s_{1}-s_{2} \in \operatorname{span}\{u\}$. Further let $f_{+}^{\prime}\left(x_{0}, q\right)$ exist for each $q \in X$ and

$$
\begin{gathered}
\left|\frac{f(x+t u)-f(x)}{t}-U\right| \leq \varepsilon,\left|\frac{f(x+t v)-f(x)}{t}-V\right| \leq \varepsilon, \\
\left|\frac{f(x+t w)-f(x)}{t}-W\right| \leq \varepsilon
\end{gathered}
$$

whenever $x \in M$ and $0<t<\delta$. Then $s_{1}=s_{2}$.

Proof. Suppose on the contrary that $s_{1} \neq s_{2}$. Then we can suppose without any loss of generality that $s_{1}-s_{2}=\alpha u$, where $\alpha>0$. By Lemma 3.1 (i) there exist vectors $\varepsilon_{1}, \varepsilon_{2}, \varepsilon_{3} \in Y,\left\|\varepsilon_{i}\right\| \leq \varepsilon, i=1,2,3$, such that

$$
\begin{aligned}
f_{+}^{\prime}\left(x_{0}, s_{1}+\alpha v\right) & =f_{+}^{\prime}\left(x_{0}, s_{1}\right)+\alpha V+\alpha \varepsilon_{1}, \\
f_{+}^{\prime}\left(x_{0}, s_{2}+\alpha w\right) & =f_{+}^{\prime}\left(x_{0}, s_{2}\right)+\alpha W+\alpha \varepsilon_{2}, \\
f_{+}^{\prime}\left(x_{0}, s_{1}\right)=f^{\prime}\left(x_{0}, s_{2}+\alpha u\right) & =f_{+}^{\prime}\left(x_{0}, s_{2}\right)+\alpha U+\alpha \varepsilon_{3} .
\end{aligned}
$$

Since $s_{1}+\alpha v=s_{2}+\alpha w$, the above equalities imply

$$
\begin{aligned}
f_{+}^{\prime}\left(x_{0}, s_{1}\right)+\alpha V+\alpha \varepsilon_{1} & =f_{+}^{\prime}\left(x_{0}, s_{2}\right)+\alpha W+\alpha \varepsilon_{2}, \\
f_{+}^{\prime}\left(x_{0}, s_{1}\right) & =f_{+}^{\prime}\left(x_{0}, s_{2}\right)+\alpha U+\alpha \varepsilon_{3} .
\end{aligned}
$$

Subtracting these equalities, we obtain

$$
\begin{aligned}
\alpha V+\alpha \varepsilon_{1} & =\alpha W+\alpha \varepsilon_{2}-\alpha U-\alpha \varepsilon_{3}, \\
W-(U+V) & =\varepsilon_{1}+\varepsilon_{3}-\varepsilon_{2}, \\
\|W-(U+V)\| & \leq 3 \varepsilon,
\end{aligned}
$$

which is a contradiction.

Theorem 3.3. Let $X$ be a separable Banach space, $G \subset X$ an open set, $Y$ a Banach space and $f: G \rightarrow Y$ a Lipschitz mapping. Let $S_{+}(f)$ be the set of all points $x \in G$ at which there exist all one-sided directional derivatives 
$f_{+}^{\prime}(x, v), v \in X$, but $f$ is not Gâteaux differentiable at $x$. Then $S_{+}(f)$ can be covered by countably many Lipschitz surfaces of codimension 1. More precisely, $S_{+}(f)$ is a countable union of sets from the class $\mathcal{A}_{1}^{+}$.

If, moreover, $f$ has all one-sided directional derivatives at all points of $G$, then $S_{+}(f)$ is a countable union of closed sets from the class $\mathcal{A}_{1}^{+}$.

Proof. Since the closed linear span of the range $f(G)$ is clearly separable, we may suppose without any loss of generality that $Y$ is separable. Let $D_{X}$ and $D_{Y}$ be countable dense subsets of $X$ and $Y$, respectively, and let $Q^{+}$be the set of all positive rational numbers. Put

$$
\begin{gathered}
P=\left\{(u, v, w, U, V, W, \varepsilon, \delta): u \in D_{X}, v \in D_{X}, w=u+v, U \in D_{Y},\right. \\
\left.V \in D_{Y}, W \in D_{Y}, \varepsilon \in Q^{+}, \delta \in Q^{+},\|W-(U+V)\|>3 \varepsilon\right\} .
\end{gathered}
$$

Note that $P$ is countable. For each $p=(u, v, w, U, V, W, \varepsilon, \delta) \in P$ denote by $M(p)$ the set of all $x \in G$ for which $\operatorname{dist}(x, X \backslash G) \geq \delta$ and

$$
\begin{aligned}
\left\|\frac{f(x+t u)-f(x)}{t}-U\right\| & \leq \varepsilon,\left\|\frac{f(x+t v)-f(x)}{t}-V\right\| \leq \varepsilon, \\
\left\|\frac{f(x+t w)-f(x)}{t}-W\right\| & \leq \varepsilon \text { for each } 0<t<\delta .
\end{aligned}
$$

It is easy to see that each $M(p), p \in P$, is a closed set. We have also

$$
S_{+}(f) \subset \bigcup_{p \in P} M(p) .
$$

Indeed, if $x \in S_{+}(f)$, then there exist $\widetilde{u}, \widetilde{v} \in X$ such that $f_{+}^{\prime}(x, \widetilde{u})+f_{+}^{\prime}(x, \widetilde{v}) \neq$ $f_{+}^{\prime}(x, \widetilde{u}+\widetilde{v})$. (Otherwise the mapping $v \rightarrow f_{+}^{\prime}(x, v)$ would be linear and also Lipschitz (cf. Lemma 1.3 (i)); consequently it would be a Gâteaux derivative of $f$ at $x$.) Since the function $v \rightarrow f_{+}^{\prime}(x, v)$ is continuous (Lemma 1.3 (i)), we can choose $u \in D_{X}, v \in D_{X}, w:=u+v$ and $\varepsilon \in Q^{+}$such that $\| f_{+}^{\prime}(x, u)+$ $f_{+}^{\prime}(x, v)-f_{+}^{\prime}(x, w) \|>6 \varepsilon$. Now choose $U, V, W \in D_{Y}$ for which

$$
\left\|f_{+}^{\prime}(x, u)-U\right\|<\varepsilon,\left\|f_{+}^{\prime}(x, v)-V\right\|<\varepsilon,\left\|f_{+}^{\prime}(x, w)-W\right\|<\varepsilon .
$$

Then clearly $\|W-(U+V)\|>3 \varepsilon$ and we can choose $\delta \in Q^{+}$such that $\operatorname{dist}(x, X \backslash G) \geq \delta$ and (3.1) holds. Thus $x \in M(u, v, w, U, V, W, \varepsilon, \delta)$ and (3.2) holds. Consequently $S_{+}(f)=\bigcup_{p \in P}\left(S_{+}(f) \cap M(p)\right)$. Now fix $p=$ $(u, v, w, U, V, W, \varepsilon, \delta) \in P$ and consider $M:=S_{+}(f) \cap M(p)$. Lemma 3.2 implies that $(\operatorname{contg}(M, x)-\operatorname{contg}(M, x)) \cap \operatorname{span}\{u\} \subset\{0\}$ and therefore contg $(M, x) \cap$ $\operatorname{span}\{u\}=\emptyset$ for each $x \in M$. By Lemma 2.10 there exists a sequence $\left(S_{n}\right)_{n=1}^{\infty}$ 
of Lipschitz surfaces of codimension 1 associated with span $\{u\}$ such that $M \subset$ $\bigcup_{n=1}^{\infty} S_{n}$ and therefore $M=\bigcup_{n=1}^{\infty}\left(S_{n} \cap M\right)$. Fix $n \in \mathbb{N}$ and put $M^{*}=S_{n} \cap M$. By Lemma 3.2 we have $\left(\operatorname{contg}\left(M^{*}, x\right)-\operatorname{contg}\left(M^{*}, x\right)\right) \cap \operatorname{span}\{u\} \subset\{0\}$ for each $x \in M^{*}$. Consequently Lemma 2.11 (i) implies $M^{*} \in \mathcal{A}_{1}^{+}$and therefore $S_{+}(f)$ is a countable union of sets from the class $\mathcal{A}_{1}^{+}$. Now suppose that $f$ has all one-sided derivatives at each point $x \in G$. Then we even have $S_{+}(f)=$ $\bigcup_{p \in P} M(p)$. Indeed, if $x \in M(p)$ for some $p=(u, v, w, U, V, W, \varepsilon, \delta) \in P$, we clearly obtain $\left\|f_{+}^{\prime}(x, u)-U\right\| \leq \varepsilon,\left\|f_{+}^{\prime}(x, v)-V\right\| \leq \varepsilon,\left\|f_{+}^{\prime}(x, w)-W\right\| \leq \varepsilon$. Therefore $\left\|f_{+}^{\prime}(x, u)+f_{+}^{\prime}(x, v)-f_{+}^{\prime}(x, w)\right\|>0$ and thus $x \in S_{+}(f)$. Consequently the sets $M=S_{+}(f) \cap M(p)=M(p)$ and $M^{*}=S_{n} \cap M$ considered above are closed. Therefore $S_{+}(f)$ is a countable union of closed sets from the class $\mathcal{A}_{1}^{+}$.

Remark 3.4. Note that we have proved the above theorem using Lemma 2.11 (i). In particular the above proof gives (without using Remark 2.12) that $S_{+}(f)$ can be written in the form $S_{+}(f)=\bigcup_{n=1}^{\infty} M_{n}$ where $M_{n}$ are sets for which

$$
\left(\operatorname{contg}\left(M_{n}, x\right)-\operatorname{contg}\left(M_{n}, x\right)\right) \cap \operatorname{span}\{u\} \subset\{0\}, x \in M_{n}
$$

Moreover, if $f$ has all one-sided derivatives at each point $x \in G$, then $M_{n}$ can be chosen to be closed. Further note that (3.3) clearly implies int $\left(\operatorname{contg}\left(M_{n}, x\right)\right)$ $=\emptyset, x \in M_{n}$.

Lemma 3.5. Let $M \subset X, x_{0}, u, v, w, s_{1}, s_{2} \in X, \varepsilon, \delta>0, U, V, W \in Y$ be given such that $w=u+v,\|W-(U+V)\|>5 \varepsilon, s_{1}, s_{2} \in \operatorname{contg}\left(M, x_{0}\right)$. Further let $f^{\prime}\left(x_{0}, q\right)$ exist for each $q \in X$ and

$$
\begin{gathered}
\left|\frac{f(x+t u)-f(x)}{t}-U\right| \leq \varepsilon, \quad\left|\frac{f(x+t v)-f(x)}{t}-V\right| \leq \varepsilon, \\
\left|\frac{f(x+t w)-f(x)}{t}-W\right| \leq \varepsilon,
\end{gathered}
$$

whenever $x \in M$ and $0<|t|<\delta$. Then the following assertions hold.

(i) If $s_{1}-s_{2} \in \operatorname{span}\{u, v\}$, then $s_{1}=s_{2}$.

(ii) If $s_{1}+s_{2} \in \operatorname{span}\{u, v\}$, then $s_{1}=-s_{2}$.

Proof. To prove (i), suppose on the contrary that $s_{1}-s_{2} \in \operatorname{span}\{u, v\}$ and $s_{1} \neq s_{2}$. Without any loss of generality we can suppose that $s_{1}-s_{2}=$ 
$\alpha u+\beta v$, where $|\alpha|>0$ and $|\alpha| \geq|\beta|$. By Lemma 3.1 (ii) there exist vectors $\varepsilon_{1}, \varepsilon_{2}, \varepsilon_{3}, \varepsilon_{4} \in Y,\left\|\varepsilon_{i}\right\| \leq \varepsilon, i=1,2,3,4$, such that

$$
\begin{aligned}
f^{\prime}\left(x_{0}, s_{1}-\beta v\right) & =f^{\prime}\left(x_{0}, s_{1}\right)-\beta V+|\beta| \varepsilon_{1}, \\
f^{\prime}\left(x_{0}, s_{2}+\alpha u\right) & =f^{\prime}\left(x_{0}, s_{2}\right)+\alpha U+|\alpha| \varepsilon_{2}, \\
f^{\prime}\left(x_{0}, s_{1}-\alpha w\right) & =f^{\prime}\left(x_{0}, s_{1}\right)-\alpha W+|\alpha| \varepsilon_{3}, \\
f^{\prime}\left(x_{0}, s_{2}+(\beta-\alpha) v\right) & =f^{\prime}\left(x_{0}, s_{2}\right)+(\beta-\alpha) V+|\beta-\alpha| \varepsilon_{4} .
\end{aligned}
$$

Since $s_{1}-\beta v=s_{2}+\alpha u$ and $s_{1}-\alpha w=s_{2}+(\beta-\alpha) v$, the above equalities imply

$$
\begin{aligned}
f^{\prime}\left(x_{0}, s_{1}\right)-\beta V+|\beta| \varepsilon_{1} & =f^{\prime}\left(x_{0}, s_{2}\right)+\alpha U+|\alpha| \varepsilon_{2}, \\
f^{\prime}\left(x_{0}, s_{1}\right)-\alpha W+|\alpha| \varepsilon_{3} & =f^{\prime}\left(x_{0}, s_{2}\right)+(\beta-\alpha) V+|\beta-\alpha| \varepsilon_{4} .
\end{aligned}
$$

Subtracting these equalities, we obtain

$$
\begin{aligned}
\alpha W-\beta V+|\beta| \varepsilon_{1}-|\alpha| \varepsilon_{3} & =\alpha U-(\beta-\alpha) V+|\alpha| \varepsilon_{2}-|\beta-\alpha| \varepsilon_{4}, \\
W-(U+V) & =\frac{1}{\alpha}\left(|\alpha| \varepsilon_{2}-|\beta-\alpha| \varepsilon_{4}-|\beta| \varepsilon_{1}+|\alpha| \varepsilon_{3}\right), \\
\|W-(U+V)\| & \leq \varepsilon+2 \varepsilon+\varepsilon+\varepsilon=5 \varepsilon,
\end{aligned}
$$

which is a contradiction.

To prove (ii), suppose on the contrary that $s_{1}+s_{2} \in \operatorname{span}\{u, v\}$ and $s_{1} \neq-s_{2}$. Without any loss of generality we can suppose that $s_{1}+s_{2}=$ $\alpha u+\beta v$, where $|\alpha|>0$ and $|\alpha| \geq|\beta|$. By Lemma 3.1 there exist vectors $\varepsilon_{1}, \varepsilon_{2}, \varepsilon_{3}, \varepsilon_{4} \in Y,\left\|\varepsilon_{i}\right\| \leq \varepsilon, i=1,2,3,4$, such that

$$
\begin{aligned}
f^{\prime}\left(x_{0}, s_{1}-\alpha u\right) & =f^{\prime}\left(x_{0}, s_{1}\right)-\alpha U+|\alpha| \varepsilon_{1}, \\
f^{\prime}\left(x_{0}, s_{2}-\beta v\right) & =f^{\prime}\left(x_{0}, s_{2}\right)-\beta V+|\beta| \varepsilon_{2}, \\
f^{\prime}\left(x_{0}, s_{1}-\alpha w\right) & =f^{\prime}\left(x_{0}, s_{1}\right)-\alpha W+|\alpha| \varepsilon_{3}, \\
f^{\prime}\left(x_{0}, s_{2}+(\alpha-\beta) v\right) & =f^{\prime}\left(x_{0}, s_{2}\right)+(\alpha-\beta) V+|\alpha-\beta| \varepsilon_{4} .
\end{aligned}
$$

Since $s_{1}-\alpha u=-\left(s_{2}-\beta v\right)$ and $s_{1}-\alpha w=-\left(s_{2}+(\alpha-\beta) v\right)$, the above equalities imply

$$
\begin{aligned}
f^{\prime}\left(x_{0}, s_{1}\right)-\alpha U+|\alpha| \varepsilon_{1} & =-\left(f^{\prime}\left(x_{0}, s_{2}\right)-\beta V+|\beta| \varepsilon_{2}\right), \\
f^{\prime}\left(x_{0}, s_{1}\right)-\alpha W+|\alpha| \varepsilon_{3} & =-\left(f^{\prime}\left(x_{0}, s_{2}\right)+(\alpha-\beta) V+|\alpha-\beta| \varepsilon_{4}\right) .
\end{aligned}
$$

Subtracting these equalities, we obtain

$$
\alpha W-\alpha U+|\alpha| \varepsilon_{1}-|\alpha| \varepsilon_{3}=\alpha V+|\alpha-\beta| \varepsilon_{4}-|\beta| \varepsilon_{2},
$$




$$
\begin{aligned}
W-(U+V) & =\frac{1}{\alpha}\left(-|\alpha| \varepsilon_{1}+|\alpha| \varepsilon_{3}+|\alpha-\beta| \varepsilon_{4}-|\beta| \varepsilon_{2}\right), \\
\|W-(U+V)\| & \leq \varepsilon+\varepsilon+2 \varepsilon+\varepsilon=5 \varepsilon,
\end{aligned}
$$

which is a contradiction.

Theorem 3.6. Let $X$ be a separable Banach space, $G \subset X$ an open set, $Y$ a Banach space and $f: G \rightarrow Y$ a Lipschitz mapping. Let $S(f)$ be the set of all points $x \in G$ at which there exist all (two-sided) directional derivatives $f^{\prime}(x, v), v \in X$, but $f$ is not Gâteaux differentiable at $x$. Then $S(f)$ can be covered by countably many Lipschitz surfaces of codimension 2. More precisely, $S(f)$ is a countable union of sets from the class $\mathcal{A}_{2}$. If, moreover, $f$ has all (two-sided) directional derivatives at all points of $G$, then $S(f)$ is a countable union of closed sets from the class $\mathcal{A}_{2}$.

Proof. Since the closed linear span of the range $f(G)$ is clearly separable, we may suppose without any loss of generality that $Y$ is separable. Let $D_{X}$ and $D_{Y}$ be countable dense subsets of $X$ and $Y$, respectively, and let $Q^{+}$be the set of all positive rational numbers. Put

$$
\begin{gathered}
P=\left\{(u, v, w, U, V, W, \varepsilon, \delta): u \in D_{X}, v \in D_{X}, w=u+v, U \in D_{Y},\right. \\
\left.V \in D_{Y}, W \in D_{Y}, \varepsilon \in Q^{+}, \delta \in Q^{+},\|W-(U+V)\|>5 \varepsilon\right\} .
\end{gathered}
$$

Note that $P$ is countable. For each $p=(u, v, w, U, V, W, \varepsilon, \delta) \in P$ denote by $M(p)$ the set of all $x \in G$ for which $\operatorname{dist}(x, X \backslash G) \geq \delta$ and

$$
\begin{aligned}
& \left\|\frac{f(x+t u)-f(x)}{t}-U\right\| \leq \varepsilon,\left\|\frac{f(x+t v)-f(x)}{t}-V\right\| \leq \varepsilon, \\
& \left\|\frac{f(x+t w)-f(x)}{t}-W\right\| \leq \varepsilon \text { for each } 0<|t|<\delta .
\end{aligned}
$$

It is easy to see that each $M(p), p \in P$, is a closed set. We have also

$$
S(f) \subset \bigcup_{p \in P} M(p) .
$$

Indeed, if $x \in S(f)$, then there exist $\widetilde{u}, \widetilde{v} \in X$ such that $f^{\prime}(x, \widetilde{u})+f^{\prime}(x, \widetilde{v}) \neq$ $f^{\prime}(x, \widetilde{u}+\widetilde{v})$. (Otherwise the mapping $v \rightarrow f^{\prime}(x, v)$ would be linear and also Lipschitz (cf. Lemma 1.3 (i)); consequently it would be a Gâteaux derivative of $f$ at $x$.) Since the function $v \rightarrow f^{\prime}(x, v)$ is continuous (Lemma 1.3 (i)), we can choose $u \in D_{X}, v \in D_{X}, w:=u+v$ and $\varepsilon \in Q^{+}$such that $\| f^{\prime}(x, u)+$ $f^{\prime}(x, v)-f^{\prime}(x, w) \|>8 \varepsilon$. Now choose $U, V, W \in D_{Y}$ for which

$$
\left\|f^{\prime}(x, u)-U\right\|<\varepsilon, \quad\left\|f^{\prime}(x, v)-V\right\|<\varepsilon, \quad\left\|f^{\prime}(x, w)-W\right\|<\varepsilon .
$$


Then clearly $\|W-(U+V)\|>5 \varepsilon$ and we can choose $\delta \in Q^{+}$such that $\operatorname{dist}(x, X \backslash G) \geq \delta$ and (3.4) holds. Therefore $x \in M(u, v, w, U, V, W, \varepsilon, \delta)$ and (3.5) holds. Consequently $S(f)=\bigcup_{p \in P}(S(f) \cap M(p))$. Now fix $p=$ $(u, v, w, U, V, W, \varepsilon, \delta) \in P$ and consider $M:=S(f) \cap M(p)$. Lemma 3.5 immediately implies that $(\operatorname{contg}(M, x)-\operatorname{contg}(M, x)) \cap \operatorname{span}\{u, v\} \subset\{0\}$ and therefore $\operatorname{contg}(M, x) \cap \operatorname{span}\{u, v\}=\emptyset$ for each $x \in M$. By Lemma 2.10 there exists a sequence $\left(S_{n}\right)_{n=1}^{\infty}$ of Lipschitz surfaces of codimension 2 associated with $\operatorname{span}\{u, v\}$ such that $M \subset \bigcup_{n=1}^{\infty} S_{n}$ and therefore $M=$ $\bigcup_{n=1}^{\infty}\left(S_{n} \cap M\right)$. Fix $n \in \mathbb{N}$ and put $M^{*}=S_{n} \cap M$. By Lemma 3.5 we have $\left(\operatorname{contg}\left(M^{*}, x\right)-\operatorname{contg}\left(M^{*}, x\right)\right) \cap \operatorname{span}\{u, v\} \subset\{0\},\left(\operatorname{contg}\left(M^{*}, x\right)+\right.$ $\left.\operatorname{contg}\left(M^{*}, x\right)\right) \cap \operatorname{span}\{u, v\} \subset\{0\}$ for all $x \in M^{*}$. Consequently Lemma 2.11 (ii) implies $M^{*} \in \mathcal{A}_{2}$ and therefore $S(f)$ is a countable union of sets from the class $\mathcal{A}_{2}$. Now suppose that $f$ has all two-sided derivatives at each point $x \in G$. Then we have even $S(f)=\bigcup_{p \in P} M(p)$. Indeed, if $x \in M(p)$ for some $p=(u, v, w, U, V, W, \varepsilon, \delta) \in P$, we clearly obtain $\left\|f^{\prime}(x, u)-U\right\| \leq \varepsilon, \| f^{\prime}(x, v)-$ $V\|\leq \varepsilon,\| f^{\prime}(x, w)-W \| \leq \varepsilon$. Therefore $\left\|f^{\prime}(x, u)+f^{\prime}(x, v)-f^{\prime}(x, w)\right\|>2 \varepsilon$ and thus $x \in S(f)$. Consequently the sets $M=S(f) \cap M(p)=M(p)$ and $M^{*}=S_{n} \cap M$ considered above are closed. Therefore $S(f)$ is a countable union of closed sets from the class $\mathcal{A}_{2}$.

\section{Constructions}

In the following lemma we use the method of B. Kirchheim's proof of Proposition 14 from [PZ]. Recall the notation $B(x, r)$ for the open ball with a center $x$ and radius $r$.

Lemma 4.1. Let $X$ be a separable Banach space and $f$ a non-negative bounded Lipschitz function defined on $X$. Let $F:=\{x: f(x)=0\} \neq \emptyset$. Then there exists a function $g$ such that

(i) $g \uparrow_{F}=0, g \uparrow_{(X \backslash F)}>0$;

(ii) $g$ is Gâteaux differentiable on $X \backslash F$;

(iii) $g$ is Lipschitz ;

(iv) the Fréchet derivative of $f-g$ is 0 at each point of $F$.

Proof. Assume first $0 \leq f \leq 1, \operatorname{Lip}(f) \leq 1$. Set

$$
f_{k}(x)=\min \left(\max \left(f(x)-2^{-k}, 0\right), 2^{-k}\right), k \in \mathbb{N} .
$$


It is easy to see that $f=\sum_{k=1}^{\infty} f_{k}, 0 \leq f_{k} \leq 2^{-k}, \operatorname{Lip}\left(f_{k}\right) \leq 1$ and

$$
f_{k}(x)=0 \text { if } f(x) \leq 2^{-k} ; f_{k}(x)=2^{-k} \text { if } f(x) \geq 2^{-k+1} .
$$

For each $k \in \mathbb{N}$, let $\mu_{k}$ be a non-degenerate probability cube measure (see [BL], p. 142) with

$$
\operatorname{supp} \mu_{k} \subset B\left(0,4^{-1-k}\right)
$$

and $g_{k}=f_{k} \star \mu_{k}$ (i.e. $\left.g_{k}(x)=\int f_{k}(x-t) \mathrm{d} \mu_{k}(t)\right)$. Since $f_{k}$ is Lipschitz, it is Gâteaux differentiable at $\mu_{k}$-almost all points of $X$ (see [BL], 6.25, 6.27, 6.42) and therefore Lebesque's dominated convergence theorem easily gives (as in $[\mathrm{BL}], 6.43)$ that $g_{k}^{\prime}(x, v)=\left(f_{k}^{\prime}(., v) \star \mu_{k}\right)(x)$ for every $x, v \in X$ and therefore $g_{k}$ is an everywhere Gâteaux differentiable function. It is easy to verify (using $0 \leq f_{k} \leq 2^{-k}, \operatorname{Lip}\left(f_{k}\right) \leq 1$ and (4.2)) that

$$
0 \leq g_{k} \leq 2^{-k}, \operatorname{Lip}\left(g_{k}\right) \leq 1 \text { and }\left|g_{k}-f_{k}\right| \leq 4^{-1-k} .
$$

Using (4.1), (4.2) and $\operatorname{Lip}(f) \leq 1$, we easily obtain that

$$
g_{k}(x)=f_{k}(x)=0 \text { if } f(x)<2^{-k}-4^{-1-k}
$$

and

$$
g_{k}(x)=f_{k}(x)=2^{-k} \text { if } f(x)>2^{-k+1}+4^{-1-k} .
$$

Set $g:=\sum_{k=1}^{\infty} g_{k}$. From (4.4), (4.3) and (4.5) we immediately obtain (i).

Now, fix $n \in \mathbb{N}$ and consider $g$ on the set $D_{n}=\left\{x: 2^{-n}<f(x)<2^{-n+2}\right\}$. By (4.4) and (4.5) we have that $g_{k}(x)=f_{k}(x)=0$ for $x \in D_{n}$ and $1 \leq k \leq$ $n-3 ; g_{k}(x)=f_{k}(x)=2^{-k}$ for $x \in D_{n}$ and $k \geq n+2$. Consequently,

$$
g(x)=\sum_{k \geq n+2} 2^{-k}+\sum_{\max (1, n-2) \leq k \leq n+1} g_{k}(x), \quad x \in D_{n} .
$$

Therefore $g$ is Gâteaux differentiable on $D_{n}$ and $\operatorname{Lip}(g, x) \leq 4$ for each $x \in D_{n}$. Since clearly $X \backslash F=\bigcup_{n=1}^{\infty} D_{n}$, we obtain (ii) and

$$
\operatorname{Lip}(g, x) \leq 4 \text { for each } x \in X \backslash F .
$$

Using (4.3) we obtain, for each $x \in D_{n}$,

$$
|f(x)-g(x)| \leq \sum_{\max (1, n-2) \leq k \leq n+1}\left|f_{k}(x)-g_{k}(x)\right| \leq 4 \cdot 4^{-n+1} .
$$


Fix $y \in F$ and consider $x \in X, x \neq y$. Consider for $x \in X \backslash F$ the least $n=n(x)$ for which $x \in D_{n}$. Since $\operatorname{Lip}(f) \leq 1$, we have $\|y-x\|>2^{-n}$. By (4.7) we obtain

$$
\frac{|f(x)-g(x)|}{\|y-x\|} \leq \frac{4 \cdot 4^{-n+1}}{2^{-n}} .
$$

Clearly $\lim _{\substack{x \rightarrow y \\ x \notin F}} n(x)=\infty$, which proves (iv).

Now, (iv) and $\operatorname{Lip}(f) \leq 1$ gives $\operatorname{Lip}(g, x) \leq 4$ for each $x \in F$. Using (4.6) and Lemma 1.3 (ii), we obtain (iii).

In the general case find $\varepsilon>0$ such that $\varepsilon f \leq 1$ and $\operatorname{Lip}(\varepsilon f) \leq 1$. Applying the construction above to $\widetilde{f}:=\varepsilon f$ we obtain a function $\widetilde{g}$ and $g:=\frac{1}{\varepsilon} \widetilde{g}$ satisfies the properties (i)-(iv).

The next lemma generalizes Lemma 4.1 in the sense that $F$ is any closed subset of $\{x \in X: f(x)=0\}$ but need not be equal to this set and $f$ need not be non-negative.

Lemma 4.2. Let $X$ be a separable Banach space and $f$ be a bounded Lipschitz real-valued function. Let $\emptyset \neq F \subset X$ be closed and $f \uparrow_{F}=0$. Then there exists a function $g$ such that $g$ is bounded Lipschitz, $g$ is Gâteaux differentiable on $X \backslash F,\left.g\right|_{F}=0$ and $f-g$ has zero Fréchet derivative at each point $x \in F$.

Proof. Set $d(x)=\min (\operatorname{dist}(x, F), 1)$. Let $f^{+}, f^{-}$be the positive and negative parts of $f$. Put $h_{1}(x)=f^{+}(x)+d(x), h_{2}(x)=f^{-}(x)+d(x)$. Clearly $h_{1}$, $h_{2}$ are non-negative bounded Lipschitz functions and

$$
F=\left\{x: h_{1}(x)=0\right\}=\left\{x: h_{2}(x)=0\right\} .
$$

By Lemma 4.1 we can find bounded Lipschitz functions $g_{1}, g_{2}$ which are Gâteaux differentiable on $X \backslash F,\left.g_{i}\right|_{F}=0$ and $h_{i}-g_{i}$ has the Fréchet derivative 0 at each point $x \in F, i=1,2$. Set $g=g_{1}-g_{2}$. Then $g \uparrow_{F}=0$ and $g$ is Gâteaux differentiable on $X \backslash F$. Let $x \in F$. Since $f=f^{+}-f^{-}=h_{1}-h_{2}$, we have

$$
\begin{aligned}
& \frac{|(f-g)(y)-(f-g)(x)|}{\|y-x\|} \\
& \leq \frac{\left|\left(h_{1}-g_{1}\right)(y)-\left(h_{1}-g_{1}\right)(x)\right|}{\|y-x\|}+\frac{\left|\left(h_{2}-g_{2}\right)(y)-\left(h_{2}-g_{2}\right)(x)\right|}{\|y-x\|},
\end{aligned}
$$

which with the fact that $h_{i}-g_{i}$ has zero Fréchet derivative at all points of $F$ finishes the proof.

The following easy lemma is well-known (cf. Proposition 3.6. (i) from [Sp]). 
Lemma 4.3. Let $X, Y, Z$ be Banach spaces, $f: X \rightarrow Y, g: Y \rightarrow Z$ be two mappings and let $g$ be Lipschitz. Let $x, u \in X$ and let there exist $v:=f_{+}^{\prime}(x, u)$, $g_{+}^{\prime}(f(x), v)$. Then

$$
(g \circ f)_{+}^{\prime}(x, u)=g_{+}^{\prime}(f(x), v) .
$$

Remark 4.4. The preceding lemma clearly holds also in the trivial cases when at least one of the spaces $X, Y, Z$ is the trivial null-dimensional space $\{0\}$. Of course, then the directional derivatives involved in (4.8) equal to zero.

Lemma 4.5. Let $X$ be a separable Banach space and $\emptyset \neq A \in \mathcal{B}_{1}^{+}(X)$. Then there exists a bounded Lipschitz function $f: X \rightarrow \mathbb{R}$ such that

(i) $f_{+}^{\prime}(x, u)$ exists for all $x \in A$ and $u \in X$;

(ii) $f$ is Gâteaux differentiable on $X \backslash \bar{A}$;

(iii) there is $w \in X,\|w\|=1$, such that for all $x \in A, f_{+}^{\prime}(x, w)=f_{+}^{\prime}(x,-w)=$ 1.

In particular, each closed set from $\mathcal{B}_{1}^{+}(X)$ belongs to $\widetilde{\mathcal{S}}_{+}(X)$.

Proof. According to Definition 2.3 there exist a subspace $Y$ of codimension 1, a topological complement $W$ of $Y$ and a Lipschitz function $h: Y \rightarrow W$ such that $A \subset S:=\{y+h(y): y \in Y\}$ and

$$
h_{+}^{\prime}(y, v) \text { exists whenever } v \in Y \text { and } y \in P(A),
$$

where $P$ is the projection of $X$ on $Y$ parallel to $W$. For $x \in X$, put

$$
g(x):=\|x-P(x)-h(P(x))\| .
$$

Clearly $g \uparrow_{S}=0$. Since the sum and the composition of two Lipschitz functions is Lipschitz and the functions $P, h,\|$.$\| are Lipschitz, we obtain that g$ is Lipschitz. Since $P$ is linear and $\|$.$\| is convex, (4.9) and Lemma 4.3$ imply that

$$
g_{+}^{\prime}(x, u) \text { exists for each } x \in A \text { and } u \in X .
$$

Choose a unit vector $w \in W$ and suppose that $x \in A$ is given. Since clearly $P(x)=P(x+t w)$ for each $t \in \mathbb{R}$, we have

$$
g(x+t w)=\|x+t w-P(x)-h(P(x))\|=\|t w\|=|t| .
$$

Thus

$$
g_{+}^{\prime}(x, w)=1 \text { and } g_{+}^{\prime}(x,-w)=1
$$


Set $g_{1}(x):=\min (g(x), 1)$. Then $g_{1}$ satisfies the assumptions of Lemma 4.2 with $F=\bar{A}$ and so we can find a bounded Lipschitz function $f$ such that $f\left\lceil_{\bar{A}}=0\right.$, $f$ is Gâteaux differentiable on $X \backslash \bar{A}$ and $f-g_{1}$ has zero Fréchet derivative at any point $x \in \bar{A}$. These properties of $f,(4.10)$ and (4.11) immediately give (i) and (iii).

Lemma 4.6. Let $X$ be a separable Banach space, $\left\{a_{n}\right\},\left\{b_{n}\right\}$ sequences of positive numbers, $\sum_{n=1}^{\infty} a_{n}<\infty, \sum_{n=1}^{\infty} b_{n}<\infty$. Let $f_{n}: X \rightarrow \mathbb{R}$ satisfy $\left|f_{n}\right| \leq a_{n}$ and $\operatorname{Lip}\left(f_{n}\right) \leq b_{n}$. Let $\left(f_{n}\right)_{+}^{\prime}(x, u)$ exist for some $x, u \in X$. Set $f=\sum_{n=1}^{\infty} f_{n}$. Then $f$ is a Lipschitz function and

$$
f_{+}^{\prime}(x, u)=\sum_{n=1}^{\infty}\left(f_{n}\right)_{+}^{\prime}(x, u)
$$

Moreover, if $f_{n}$ are Gâteaux differentiable at $x$, then $f$ is Gâteaux differentiable at $x$.

Proof. It is easy to see that $f$ is Lipschitz. For $t \in(0, \infty)$, put

$$
g(t)=\frac{1}{t}(f(x+t u)-f(x)), g_{n}(t)=\frac{1}{t}\left(f_{n}(x+t u)-f_{n}(x)\right) .
$$

Clearly, $g(t)=\sum_{n=1}^{\infty} g_{n}(t)$ for $t>0$, and $\left|g_{n}(t)\right| \leq b_{n}\|u\|$ because $\operatorname{Lip}\left(f_{n}\right) \leq b_{n}$. Consequently $\sum_{n=1}^{\infty} g_{n}(t)$ converges uniformly on $(0, \infty)$. Therefore

$$
\sum_{n=1}^{\infty}\left(f_{n}\right)_{+}^{\prime}(x, u)=\sum_{n=1}^{\infty} \lim _{t \rightarrow 0_{+}} g_{n}(t)=\lim _{t \rightarrow 0_{+}} g(t)=f_{+}^{\prime}(x, u),
$$

which proves (4.12).

If $f_{n}$ are Gâteaux differentiable at $x$, then the mappings $u \rightarrow\left(f_{n}\right)_{+}^{\prime}(x, u)$ are linear on $X$. By (4.12) we have that $v \rightarrow f_{+}^{\prime}(x, v)$ is linear and it is also continuous by Lemma 1.3 (i).

Theorem 4.7. Let $X$ be a separable Banach space, $\operatorname{dim}(X) \geq 1$, and $A \subset X$ be a countable union of closed sets from $\mathcal{B}_{1}^{+}$. Then there exists a Lipschitz function $f: X \rightarrow \mathbb{R}$ such that $f_{+}^{\prime}(x, u)$ exists for all $x, u \in X, f$ is Gâteaux differentiable at all points of $X \backslash A$ and $f$ is Gâteaux differentiable at no point of $A$ (i.e. $A \in \widetilde{\mathcal{S}}_{+}(X)$ ). 
Proof. The case $A=\emptyset$ is trivial. Thus suppose that $A=\bigcup_{n=1}^{\infty} A_{n}$, where $\emptyset \neq A_{n} \in \mathcal{B}_{1}^{+}$are closed. According to Lemma 4.5 there are vectors $w_{n} \in X$, $\left\|w_{n}\right\|=1$, functions $f_{n}: X \rightarrow \mathbb{R}$ and numbers $L_{n}, K_{n}$ with

$$
\begin{aligned}
& \left|f_{n}\right| \leq L_{n}, \operatorname{Lip}\left(f_{n}\right) \leq K_{n} ; \\
& \left(f_{n}\right)_{+}^{\prime}(x, u) \text { exists for all } x, u \in X ; \\
& f_{n} \text { is Gâteaux differentiable on } X \backslash A_{n} ; \\
& \left(f_{n}\right)_{+}^{\prime}\left(x, w_{n}\right)=\left(f_{n}\right)_{+}^{\prime}\left(x,-w_{n}\right)=1 \text { for all } x \in A_{n} .
\end{aligned}
$$

Without loss of generality we can suppose $L_{n} \geq 1, K_{n} \geq 1$. Define a sequence $\left\{\varepsilon_{n}\right\}$ by

$$
\varepsilon_{1}=\frac{1}{L_{1} K_{1}}, \varepsilon_{n+1}=\frac{\varepsilon_{n}}{3 L_{n+1} K_{n+1}} .
$$

Since $L_{n} \geq 1, K_{n} \geq 1$, we have

$$
\varepsilon_{n} \leq 3^{l-n} \varepsilon_{l} \text { for } 1 \leq l \leq n
$$

and consequently, for each $n>1, L_{n} \varepsilon_{n}=\left(3 K_{n}\right)^{-1} \varepsilon_{n-1} \leq 3^{-1} \varepsilon_{n-1} \leq 3^{1-n} \varepsilon_{1}$. Analogously, $K_{n} \varepsilon_{n} \leq 3^{1-n} \varepsilon_{1}$. Set $g_{n}=\varepsilon_{n} f_{n}, n \in \mathbb{N}$. Then by (4.13) we have $\left|g_{n}\right| \leq 3^{1-n} \varepsilon_{1}$ and $\left|\operatorname{Lip}\left(g_{n}\right)\right| \leq 3^{1-n} \varepsilon_{1}$. Put $f=\sum_{n=1}^{\infty} g_{n}$. By Lemma 4.6, (4.14) and (4.15) we have that $f$ is Lipschitz, $f_{+}^{\prime}(x, u)$ exists for all $x, u \in X$,

$$
f_{+}^{\prime}(x, u)=\sum_{n=1}^{\infty}\left(g_{n}\right)_{+}^{\prime}(x, u) \text { for all } x, u \in X
$$

and $f$ is Gâteaux differentiable at all points of $X \backslash A$. Let $x \in A$ be given. Put $m:=\min \left\{n: x \in A_{n}\right\}$. Then by (4.15), for each $1 \leq l<m, f_{l}$ is Gâteaux differentiable at $x$ and consequently $\left(f_{l}\right)_{+}^{\prime}\left(x, w_{m}\right)+\left(f_{l}\right)_{+}^{\prime}\left(x,-w_{m}\right)=0$, which gives with (4.19) and (4.16)

$$
\begin{aligned}
f_{+}^{\prime}\left(x, w_{m}\right)+f_{+}^{\prime}\left(x,-w_{m}\right) & =\sum_{n=1}^{\infty} \varepsilon_{n}\left(\left(f_{n}\right)_{+}^{\prime}\left(x, w_{m}\right)+\left(f_{n}\right)_{+}^{\prime}\left(x,-w_{m}\right)\right) \\
& =2 \varepsilon_{m}+\sum_{n=m+1}^{\infty} \varepsilon_{n}\left(\left(f_{n}\right)_{+}^{\prime}\left(x, w_{m}\right)+\left(f_{n}\right)_{+}^{\prime}\left(x,-w_{m}\right)\right) .
\end{aligned}
$$

By (4.13) we have $\left|\left(f_{n}\right)_{+}^{\prime}(x, u)\right| \leq K_{n}$ for each $u \in X$ which gives with (4.17) 
and (4.18)

$$
\begin{array}{r}
\left|\sum_{n=m+1}^{\infty} \varepsilon_{n}\left(\left(f_{n}\right)_{+}^{\prime}\left(x, w_{m}\right)+\left(f_{n}\right)_{+}^{\prime}\left(x,-w_{m}\right)\right)\right| \leq\left|\sum_{n=m+1}^{\infty} \varepsilon_{n} 2 K_{n}\right| \\
=2 \sum_{n=m+1}^{\infty} \frac{\varepsilon_{n-1}}{3 L_{n}} \leq 2 \sum_{n=m}^{\infty} \frac{\varepsilon_{n}}{3} \leq \frac{2}{3} \sum_{n=m}^{\infty} \varepsilon_{m} 3^{m-n}=\varepsilon_{m} .
\end{array}
$$

Consequently, $f_{+}^{\prime}\left(x, w_{m}\right)+f_{+}^{\prime}\left(x,-w_{m}\right) \geq 2 \varepsilon_{m}-\varepsilon_{m}>0$, which proves the theorem.

Lemma 4.8. Let $W=\operatorname{span}\left\{w_{1}, w_{2}\right\}$ be a 2-dimensional Banach space. Then there exists a bounded Lipschitz function $q: W \rightarrow \mathbb{R}$ such that $q(0)=0$, $q^{\prime}(0, w)$ exists for each $w \in W, q^{\prime}\left(0, w_{1}\right)=q^{\prime}\left(0, w_{2}\right)=0$ and $q^{\prime}\left(0, w_{1}+w_{2}\right)=1$.

Proof. Let $L: W \rightarrow \mathbb{R}^{2}$ be the linear mapping for which $L\left(w_{1}\right)=(1,0)$ and $L\left(w_{2}\right)=(0,1)$. For $(x, y) \in \mathbb{R}^{2}$ put

$$
p(x, y)= \begin{cases}\min (x, y, 1) & x>0, y>0 \\ \max (x, y,-1) & x<0, y<0 \\ 0 & x y \leq 0\end{cases}
$$

It is easy to verify that $p$ is a bounded Lipschitz function, $p^{\prime}((0,0), w)$ exists for each $w \in \mathbb{R}^{2}, p^{\prime}((0,0),(1,0))=p^{\prime}((0,0),(0,1))=0$ and $p^{\prime}((0,0),(1,1))=1$. The function $q:=p \circ L$ has clearly all desired properties.

Lemma 4.9. Let $X$ be a separable Banach space and $A \in \mathcal{B}_{2}(X)$. Then there exists a bounded Lipschitz function $f: X \rightarrow \mathbb{R}$ such that

(i) $f^{\prime}(x, u)$ exists for all $x \in A$ and $u \in X$;

(ii) $f$ is Gâteaux differentiable on $X \backslash \bar{A}$;

(iii) there are unit vectors $w_{1}, w_{2} \in X$ such that, for each $x \in A, f^{\prime}\left(x, w_{1}\right)=$ $f^{\prime}\left(x, w_{2}\right)=0, f^{\prime}\left(x, w_{1}+w_{2}\right)=1$.

In particular, each closed set from $\mathcal{B}_{2}(X)$ belongs to $\widetilde{\mathcal{S}}(X)$.

Proof. According to Definition 2.3 there exists a closed subspace $Y$ of $X$, its topological complement $W$ of dimension 2 and a Lipschitz mapping $h: Y \rightarrow W$ such that $A \subset S:=\{y+h(y): y \in Y\}$ and

$$
h^{\prime}(y, v) \text { exists whenever } y \in P(A) \text { and } v \in Y,
$$


where $P$ is the projection of $X$ onto $Y$ parallel to $W$. Let $W=\operatorname{span}\left\{w_{1}, w_{2}\right\}$, $\left\|w_{1}\right\|=\left\|w_{2}\right\|=1$ and $q: W \rightarrow \mathbb{R}$ be the Lipschitz function from Lemma 4.8. For $x \in X$, put $g(x):=q(x-P(x)-h(P(x)))$. It is easy to see that $g$ is a bounded Lipschitz function. Since clearly $x-P(x)-h(P(x))=0$ for $x \in A$, (4.20), properties of $q$ and Lemma 4.3 easily gives that $g^{\prime}(x, v)$ exists for each $x \in$ $A$ and $v \in X$. Let $x \in A$ and $w \in W$. Then clearly $P(x+w)=P(x)$ and thus $g(x+w)=q(w)$. This fact immediately implies $g^{\prime}\left(x, w_{1}\right)=g^{\prime}\left(x, w_{2}\right)=$ $0, g^{\prime}\left(x, w_{1}+w_{2}\right)=1$. By Lemma 4.2 there exists a bounded Lipschitz function $f$ on $X$ such that $f / \bar{A}=0, f$ is Gâteaux differentiable on $X \backslash \bar{A}$ and $f^{\prime}(x, u)=g^{\prime}(x, u)$ for all $x \in A, u \in X$. Consequently $f$ has all desired properties.

Theorem 4.10. Let $X, \operatorname{dim}(X) \geq 2$, be a separable Banach space and $A \subset X$ be a countable union of closed sets from $\mathcal{B}_{2}(X)$. Then there exists a Lipschitz function $f: X \rightarrow \mathbb{R}$ such that $f^{\prime}(x, u)$ exists for all $x, u \in X, f$ is Gâteaux differentiable at all points of $X \backslash A$ and $f$ is Gâteaux differentiable at no point of $A$ (i.e. $A \in \widetilde{\mathcal{S}}(X))$.

Proof. The case $A=\emptyset$ is trivial. Thus suppose that $A=\bigcup_{n=1}^{\infty} A_{n}$, where $\emptyset \neq A_{n} \in \mathcal{B}_{2}$ are closed sets. According to Lemma 4.9 there are unit vectors $w_{1 n} \in X, w_{2 n} \in X$, functions $f_{n}: X \rightarrow \mathbb{R}$ and numbers $L_{n}, K_{n}$ with

$$
\begin{aligned}
& \left|f_{n}\right| \leq L_{n}, \operatorname{Lip}\left(f_{n}\right) \leq K_{n} ; \\
& \left(f_{n}\right)^{\prime}(x, u) \text { exists for all } x, u \in X ; \\
& f_{n} \text { is Gâteaux differentiable on } X \backslash A_{n} ; \\
& \left(f_{n}\right)^{\prime}\left(x, w_{1 n}\right)=\left(f_{n}\right)^{\prime}\left(x, w_{2 n}\right)=0, \\
& f^{\prime}\left(x, w_{1 n}+w_{2 n}\right)=1 \text { for all } x \in A_{n} .
\end{aligned}
$$

Without any loss of generality we can assume $L_{n} \geq 1, K_{n} \geq 1$. Define the sequence $\left\{\varepsilon_{n}\right\}$ by

$$
\varepsilon_{1}=\frac{1}{L_{1} K_{1}}, \quad \varepsilon_{n+1}=\frac{\varepsilon_{n}}{5 L_{n+1} K_{n+1}} .
$$

Since $L_{n} \geq 1, K_{n} \geq 1$, we have

$$
\varepsilon_{n} \leq 5^{l-n} \varepsilon_{l} \text { for } 1 \leq l \leq n
$$

and consequently, for $n>1, L_{n} \varepsilon_{n}=\left(5 K_{n}\right)^{-1} \varepsilon_{n-1} \leq 5^{-1} \varepsilon_{n-1} \leq 5^{1-n} \varepsilon_{1}$. Analogously, $K_{n} \varepsilon_{n} \leq 5^{1-n} \varepsilon_{1}$. Put $g_{n}=\varepsilon_{n} f_{n}$. Then by (4.21) we have $\left|g_{n}\right| \leq$ 
$5^{1-n} \varepsilon_{1}$ and $\left|\operatorname{Lip}\left(g_{n}\right)\right| \leq 5^{1-n} \varepsilon_{1}$. Put $f=\sum_{n=1}^{\infty} g_{n}$. By Lemma 4.6, (4.22) and (4.23) we obtain that $f$ is Lipschitz, $f^{\prime}(x, u)$ exists for all $x, u \in X$,

$$
f^{\prime}(x, u)=\sum_{n=1}^{\infty}\left(g_{n}\right)^{\prime}(x, u) \text { for all } x, u \in X
$$

and $f$ is Gâteaux differentiable at all points of $X \backslash A$. Let $x \in A$ be given. Put $m:=\min \left\{n: x \in A_{n}\right\}$. Then by (4.23), for each $1 \leq l<m, f_{l}$ is Gâteaux differentiable at $x$ and thus $\left(f_{l}\right)^{\prime}\left(x, w_{1 m}+w_{2 m}\right)=\left(f_{l}\right)^{\prime}\left(x, w_{1 m}\right)+\left(f_{l}\right)^{\prime}\left(x, w_{2 m}\right)$, which gives by (4.27)

$$
\begin{aligned}
& f^{\prime}\left(x, w_{1 m}+w_{2 m}\right)-f^{\prime}\left(x, w_{1 m}\right)-f^{\prime}\left(x, w_{2 m}\right) \\
= & \sum_{n=1}^{\infty} \varepsilon_{n}\left(\left(f_{n}\right)^{\prime}\left(x, w_{1 m}+w_{2 m}\right)-\left(f_{n}\right)^{\prime}\left(x, w_{1 m}\right)-\left(f_{n}\right)^{\prime}\left(x, w_{2 m}\right)\right) \\
= & \sum_{n=m}^{\infty} \varepsilon_{n}\left(\left(f_{n}\right)^{\prime}\left(x, w_{1 m}+w_{2 m}\right)-\left(f_{n}\right)^{\prime}\left(x, w_{1 m}\right)-\left(f_{n}\right)^{\prime}\left(x, w_{2 m}\right)\right) \\
= & \varepsilon_{m}\left(\left(f_{m}\right)^{\prime}\left(x, w_{1 m}+w_{2 m}\right)-\left(f_{m}\right)^{\prime}\left(x, w_{1 m}\right)-\left(f_{m}\right)^{\prime}\left(x, w_{2 m}\right)\right) \\
& +\sum_{n=m+1}^{\infty} \varepsilon_{n}\left(\left(f_{n}\right)^{\prime}\left(x, w_{1 m}+w_{2 m}\right)-\left(f_{n}\right)^{\prime}\left(x, w_{1 m}\right)-\left(f_{n}\right)^{\prime}\left(x, w_{2 m}\right)\right) .
\end{aligned}
$$

According to (4.24), (4.21), (4.25) and (4.26) we have

$$
\begin{aligned}
& \left|f^{\prime}\left(x, w_{1 m}+w_{2 m}\right)-f^{\prime}\left(x, w_{1 m}\right)-f^{\prime}\left(x, w_{2 m}\right)\right| \\
& \geq \varepsilon_{m}-3 \sum_{n=m+1}^{\infty} \varepsilon_{n} K_{n} \geq \varepsilon_{m}-3 \sum_{n=m+1}^{\infty} \frac{\varepsilon_{n-1}}{5 L_{n}} \\
& \geq \varepsilon_{m}-\frac{3}{5} \sum_{n=m}^{\infty} \varepsilon_{n} \geq \varepsilon_{m}-\frac{3}{5} \sum_{n=m}^{\infty} \frac{\varepsilon_{m}}{5^{n-m}}=\frac{\varepsilon_{m}}{4}>0 .
\end{aligned}
$$

Thus, $f$ is not Gâteaux differentiable at $x$ which finishes the proof.

\section{Consequences of the Theorems}

In this section we present some consequences of the above four theorems. Remember that we consider Lipschitz curves of special kind - see Definition 2.3 .

Proposition 5.1. Let $A \subset \mathbb{R}^{2}$. Then the following conditions are equivalent. 
(i) There exists a Lipschitz real function $f$ on $\mathbb{R}^{2}$ such that $f_{+}^{\prime}(x, u)$ exists for all $x, u \in \mathbb{R}^{2}$ and $A$ is the set of all Gâteaux non-differentiability points of $f$.

(ii) There exists a Banach space $Y$ and a Lipschitz function $f: \mathbb{R}^{2} \rightarrow Y$ such that $f_{+}^{\prime}(x, u)$ exists for all $x, u \in \mathbb{R}^{2}$ and $A$ is the set of all Gâteaux non-differentiability points of $f$.

(iii) $A=\bigcup_{n=1}^{\infty} F_{n}$, where each $F_{n}$ is a closed subset of a unilaterally smooth Lipschitz curve $L_{n}$.

(iv) $A$ is an $F_{\sigma}$ set which can be covered by countably many unilaterally smooth Lipschitz curves.

Proof. The implication (i) $\Rightarrow($ ii) is trivial. The implication (ii) $\Rightarrow($ iii) immediately follows from Theorem 3.3 and Lemma 2.8. The implication (iii) $\Rightarrow$ (i) immediately follows from Theorem 4.7. The conditions (iii) and (iv) are clearly equivalent.

Remark 5.2. Theorem 3.3 and Theorem 4.7 easily imply that $\widetilde{\mathcal{S}}_{+}(\mathbb{R})=\mathcal{S}_{+}(\mathbb{R})$ is the system of all countable subsets of $\mathbb{R}$, which is an easy and well-known fact.

Proposition 5.3. Let $X=\mathbb{R}^{2}$ or $X=\mathbb{R}^{3}$ and $A \subset X$. Then the following conditions are equivalent.

(i) There exists a Lipschitz real function $f$ on $X$ such that $f^{\prime}(x, u)$ exists for all $x, u \in X$ and $A$ is the set of all Gâteaux non-differentiability points of $f$.

(ii) There exists a Banach space $Y$ and a Lipschitz function $f: X \rightarrow Y$ such that $f^{\prime}(x, u)$ exists for all $x, u \in X$ and $A$ is the set of all Gâteaux non-differentiability points of $f$.

(iii) $A$ is a countable union of closed sets from $\mathcal{B}_{2}(X)$.

In the case $X=\mathbb{R}^{2}$ these conditions are equivalent to

(iv) 1 A is a countable subset of $\mathbb{R}^{2}$

and in the case $X=\mathbb{R}^{3}$ to the condition

(iv) $)_{2} A$ is an $F_{\sigma}$ set which can be covered by countably many smooth Lipschitz curves. 
Proof. The implication (i) $\Rightarrow$ (ii) is trivial. The implication (ii) $\Rightarrow$ (iii) immediately follows from Theorem 3.6, Lemma 2.9 and Remark 2.4 (i). The implication (iii) $\Rightarrow$ (i) immediately follows from Theorem 4.10. The equivalence of (iii) and (iv) ${ }_{1}$ in the case $X=\mathbb{R}^{2}$ is obvious. The equivalence of (iii) and (iv) $)_{2}$ easily follows from Lemma 2.9 .

The above propositions give immediately the following partial answers to Problems A, B (see Remark 5.2 for the case $X=\mathbb{R}$ ).

Proposition 5.4. $\widetilde{\mathcal{S}}_{+}\left(\mathbb{R}^{2}\right)$ is the system of all $F_{\sigma}$ subsets of $\mathbb{R}^{2}$ which can be covered by countably many unilaterally smooth Lipschitz curves. $\widetilde{\mathcal{S}}\left(\mathbb{R}^{3}\right)$ is the system of all $F_{\sigma}$ subsets of $\mathbb{R}^{3}$ which can be covered by countably many smooth Lipschitz curves. $\widetilde{\mathcal{S}}\left(\mathbb{R}^{2}\right)=\mathcal{S}\left(\mathbb{R}^{2}\right)$ is the system of all countable subsets of $\mathbb{R}^{2}$.

For a class of sets $\mathcal{C}$, denote by $\mathcal{C}_{H}$ the smallest hereditary class which contains all sets from $\mathcal{C}$ (i.e. $\mathcal{C}_{H}=\{A: A \subset C$ for some $C \in \mathcal{C}\}$ ) and by $\mathcal{C}_{I}$ the $\sigma$-ideal generated by $\mathcal{C}$.

Since we are not able to solve completely Problems A and B, it is natural to consider the following problem, which could be easier.

Problem C. Let $X$ be a Banach space.

(i) Characterize the classes $\left(\widetilde{\mathcal{S}}_{+}(X)\right)_{H},\left(\mathcal{S}_{+}(X)\right)_{H},(\widetilde{\mathcal{S}}(X))_{H},(\mathcal{S}(X))_{H}$.

(ii) Characterize the $\sigma$-ideals $\left(\widetilde{\mathcal{S}}_{+}(X)\right)_{I},\left(\mathcal{S}_{+}(X)\right)_{I},(\widetilde{\mathcal{S}}(X))_{I},(\mathcal{S}(X))_{I}$.

Of course, Proposition 5.4 immediately implies that $\left(\widetilde{\mathcal{S}}_{+}\left(\mathbb{R}^{2}\right)\right)_{H}=\left(\widetilde{\mathcal{S}}_{+}\left(\mathbb{R}^{2}\right)\right)_{I}$ is the system of all subsets of $\mathbb{R}^{2}$ which can be covered by countably many unilaterally smooth Lipschitz curves and $\left(\widetilde{\mathcal{S}}\left(\mathbb{R}^{3}\right)\right)_{H}=\left(\widetilde{\mathcal{S}}\left(\mathbb{R}^{3}\right)\right)_{I}$ is the system of all subsets of $\mathbb{R}^{3}$ which can be covered by countably many smooth Lipschitz curves. (Moreover, we have that $\left(\widetilde{\mathcal{S}}_{+}(\mathbb{R})\right)_{H}=\left(\widetilde{\mathcal{S}}_{+}(\mathbb{R})\right)_{I}=\left(\mathcal{S}_{+}(\mathbb{R})\right)_{H}=$ $\left(\mathcal{S}_{+}(\mathbb{R})\right)_{I}$ is the system of all countable subsets of $\mathbb{R}$ and $\left(\widetilde{\mathcal{S}}\left(\mathbb{R}^{2}\right)\right)_{H}=\left(\widetilde{\mathcal{S}}\left(\mathbb{R}^{2}\right)\right)_{I}=$ $\left(\mathcal{S}\left(\mathbb{R}^{2}\right)\right)_{H}=\left(\mathcal{S}\left(\mathbb{R}^{2}\right)\right)_{I}$ is the system of all countable subsets of $\mathbb{R}^{2}$.)

We are not able to give a satisfactory characterization of classes $\mathcal{S}_{+}\left(\mathbb{R}^{2}\right)$, $\mathcal{S}\left(\mathbb{R}^{3}\right),\left(\mathcal{S}_{+}\left(\mathbb{R}^{2}\right)\right)_{H},\left(\mathcal{S}\left(\mathbb{R}^{3}\right)\right)_{H}$. However, we have the following result which characterizes the $\sigma$-ideals $\left(\mathcal{S}_{+}\left(\mathbb{R}^{2}\right)\right)_{I},\left(\mathcal{S}\left(\mathbb{R}^{3}\right)\right)_{I}$.

Proposition 5.5. (i) $\left(\mathcal{S}_{+}\left(\mathbb{R}^{2}\right)\right)_{I}=\left(\mathcal{A}_{1}^{+}\left(\mathbb{R}^{2}\right)\right)_{I}=\left(\mathcal{B}_{1}^{+}\left(\mathbb{R}^{2}\right)\right)_{I}$.

(ii) $\left(\mathcal{S}\left(\mathbb{R}^{3}\right)\right)_{I}=\left(\mathcal{A}_{2}\left(\mathbb{R}^{3}\right)\right)_{I}=\left(\mathcal{B}_{2}\left(\mathbb{R}^{3}\right)\right)_{I}$. 
Proof. The statement (i) immediately follows from Theorem 3.3, Lemma 2.8 (which shows that $\mathcal{A}_{1}^{+}\left(\mathbb{R}^{2}\right)=\mathcal{B}_{1}^{+}\left(\mathbb{R}^{2}\right)$ ) and Lemma 4.5 (which implies that for each $M \in \mathcal{B}_{1}^{+}(X)$ there exists a Lipschitz function on $\mathbb{R}^{2}$ such that $\left.M \subset S_{+}(f)\right)$.

Similarly (ii) follows immediately from Theorem 3.6, Lemma 2.9 and Lemma 4.9 .

We have a complete answer to Problem $\mathrm{C}$ in no other case. However, our results yield some partial answers which are based on the following proposition.

Proposition 5.6. Let $X$ be a separable Banach space and $M \subset X$ be a $G_{\delta}$ set. Then the following assertions hold.

(i) If there exists a set $D \subset \bar{M}$ dense in $\bar{M}$ such that $\operatorname{int}(\operatorname{contg}(M, x)) \neq \emptyset$ for each $x \in D$, then $M \notin\left(\widetilde{\mathcal{S}}_{+}(X)\right)_{I}$.

(ii) If $\operatorname{int}(\operatorname{contg}(M, x)) \neq \emptyset$ for each $x \in M$, then $M \notin\left(\mathcal{S}_{+}(X)\right)_{I}$.

Proof. To prove (i), suppose on the contrary that $M \in\left(\widetilde{\mathcal{S}}_{+}(X)\right)_{I}$. By Theorem 3.3 there exist closed sets $F_{n} \in \mathcal{A}_{1}^{+}, n=1,2, \ldots$, such that $M \subset \bigcup_{n=1}^{\infty} F_{n}$. Using Remark 3.4 (cf. also Remark 2.12) we have also that int $\left(\operatorname{contg}\left(F_{n}, x\right)\right)=$ $\emptyset$ for each $x \in F_{n}$. Since $M$ is a Baire space, there exists an open set $G \subset X$ and $n \in \mathbb{N}$ such that $\emptyset \neq G \cap M \subset F_{n}$. Choose a point $d \in G \cap D$. By the assumptions of (i) we have $\operatorname{int}(\operatorname{contg}(M, d)) \neq \emptyset$. Consequently also $\operatorname{int}\left(\operatorname{contg}\left(F_{n}, d\right)\right) \neq \emptyset$, which is a contradiction.

It seems that (ii) cannot be proved directly by the Baire category theorem, but a proof similar to the proof of the Baire category theorem can be given. This phenomenon is frequent in proofs which show that a "small" set is not $\sigma$ porous. Actually, we will show that (ii) is an easy consequence of well-known facts on "abstract porosity".

We will say that $A \subset X$ is $(c)$-porous at $a \in X$ if $\operatorname{int}(\operatorname{contg}(A, a))=\emptyset$. A set is said to be $(c)$-porous if it is $(c)$-porous at each its point. A set is said to be $\sigma$ - $(c)$-porous if it is a countable union of $(c)$-porous sets.

Now we will use the generalized Foran lemma for $G_{\delta}$-sets which is proved in [Z6]. It works with the notion of abstract porosity; we shall formulate this lemma here only in the special case of $(c)$-porosity. (It is clear that $(c)$-porosity satisfies axioms of "abstract porosity". Namely: it is a local notion and if $A$ is (c)-porous at $x$, then also each subset of $\bar{A}$ is porous at $x$.)

We will say that a non-empty system $\mathcal{F}$ of non-empty $G_{\delta}$-subsets of a Banach space $X$ is a generalized Foran system (for $(c)$-porosity) if the following condition holds. 
If $A \in \mathcal{F}, H$ is open and $A \cap H \neq \emptyset$, then there exists $A^{*} \in \mathcal{F}$ such that $A^{*} \subset A \cap H$ and $A \cap H$ is (c)-porous at no point of $A^{*}$.

The generalized Foran lemma (for $(c)$-porosity) is the following result.

Let $X$ be a Banach space and let $\mathcal{F}$ be a generalized Foran system of $G_{\delta}$ subsets of $X$. Then $\mathcal{F}$ contains no $\sigma-(c)$-porous set.

Now suppose that (ii) does not hold. In the above terminology, $M$ is (c)porous at no of its point and $M \in\left(\mathcal{S}_{+}(X)\right)_{I}$. Using Theorem 3.3 and Remark 3.4 (cf. also Remark 2.12) we obtain that $M$ is $\sigma$-(c)-porous. It is easy to see that $\mathcal{F}:=\{M \cap G: G$ is open and $M \cap G \neq \emptyset\}$ is a Foran system. Thus the generalized Foran lemma implies that $M$ is not $\sigma$-(c)-porous which is a contradiction.

In the subsequent proposition we will use the following notation.

If $X$ is a Banach space, then we denote by $\mathcal{L}$ the system of all Lipschitz surfaces in $X$ of codimension 1 and by $\mathcal{L}_{+}$the system of all unilaterally smooth Lipschitz surfaces in $X$ of codimension 1.

Proposition 5.7. Let $X$ be a separable Banach space, $\operatorname{dim}(X) \geq 2$. Then

$$
\left(\widetilde{\mathcal{S}}_{+}(X)\right)_{I} \subset\left(\mathcal{S}_{+}(X)\right)_{I},\left(\mathcal{L}_{+}\right)_{I} \subset\left(\mathcal{S}_{+}(X)\right)_{H},\left(\mathcal{S}_{+}(X)\right)_{I} \subset(\mathcal{L})_{I}
$$

and all these inclusions are proper.

Proof. The inclusion $\left(\widetilde{\mathcal{S}}_{+}(X)\right)_{I} \subset\left(\mathcal{S}_{+}(X)\right)_{I}$ is trivial. To prove that it is proper, by Proposition 5.6 (i) it is sufficient to find a $G_{\delta}$ set $M \in\left(\mathcal{S}_{+}(X)\right)_{H}$ and a dense subset $D$ of $\bar{M}$ such that

$$
\operatorname{int}(\operatorname{contg}(M, x)) \neq \emptyset \text { for each } x \in D .
$$

To this end find closed subspaces $W, Y, Z, V$ of $X$ such that $\operatorname{dim} W=1, X=$ $Y \oplus W, \operatorname{dim} Z=1$ and $Y=Z \oplus V$. Choose $0 \neq w \in W, 0 \neq z \in Z$ and a dense countable subset $C$ of $\mathbb{R}$. It is not difficult to construct (see the proof of Proposition 5.8 below for a more general construction) a Lipschitz function $\varphi: \mathbb{R} \rightarrow \mathbb{R}$ which is differentiable at all points of $\mathbb{R} \backslash C$ and

$$
\varphi_{+}^{\prime}(x) \text { exists at no point } x \in C .
$$

If $y \in Y$ is of the form $y=\alpha z+v$, where $\alpha \in \mathbb{R}$ and $v \in V$, put $h(y):=\varphi(\alpha) w$. Further put

$$
\begin{aligned}
M & :=\{y+h(y): y=\alpha z+v, v \in V, \alpha \in \mathbb{R} \backslash C\}, \text { and } \\
D & :=\{y+h(y): y=\alpha z+v, v \in V, \alpha \in C\} .
\end{aligned}
$$


Clearly $M$ is a $G_{\delta}$ set, $M \in \mathcal{B}_{1}(X)$ and $D$ is dense in $\bar{M}$. By Lemma 4.5 we have $M \in\left(\mathcal{S}_{+}(X)\right)_{H}$. Since it is not difficult to see that (5.2) implies (5.1), we are done.

Theorem 4.7 immediately implies the inclusion $\left(\mathcal{L}_{+}\right)_{I} \subset\left(\widetilde{\mathcal{S}}_{+}(X)\right)_{H}$. Since the inclusion $\left(\widetilde{\mathcal{S}}_{+}(X)\right)_{I} \subset\left(\mathcal{S}_{+}(X)\right)_{I}$ is proper, clearly the inclusion $\left(\widetilde{\mathcal{S}}_{+}(X)\right)_{H} \subset$ $\left(\mathcal{S}_{+}(X)\right)_{H}$ is also proper and therefore the inclusion $\left(\mathcal{L}_{+}\right)_{I} \subset\left(\mathcal{S}_{+}(X)\right)_{H}$ is proper as well.

The inclusion $\left(\mathcal{S}_{+}(X)\right)_{I} \subset(\mathcal{L})_{I}$ follows from Theorem 3.3. To show that it is proper, we will need a function $\widetilde{\varphi}: \mathbb{R} \rightarrow \mathbb{R}$ such that $\widetilde{\varphi}$ is Lipschitz and there exists a dense $G_{\delta}$ set $P \subset \mathbb{R}$ such that $\widetilde{\varphi}_{+}^{\prime}(x)$ exists at no point $x \in P$.

The existence of such a function is well-known (for a well-known easy construction, see [Br]). Now let $W, Y, Z, V, w, z, h$ be defined as above, but now using the function $\widetilde{\varphi}$ instead of $\varphi$. Put $\widetilde{M}=\{y+h(y): y=\alpha z+v, \alpha \in P, v \in$ $V\}$. Of course, $\widetilde{M}$ is a subset of a Lipschitz surface and thus $\widetilde{M} \in(\mathcal{L})_{H} \subset(\mathcal{L})_{I}$. On the other hand, $\widetilde{M}$ is clearly a $G_{\delta}$ set and it is not difficult to verify that $\operatorname{int}(\operatorname{contg}(\widetilde{M}, x)) \neq \emptyset$ for each $x \in \widetilde{M}$. Consequently Proposition 5.6 (ii) implies $\widetilde{M} \notin\left(\mathcal{S}_{+}(X)\right)_{I}$.

In the proof of the following proposition we will use the following notation. If $W$ is a Banach space, $f: \mathbb{R} \rightarrow W$ and $a \in \mathbb{R}$, then we put

$$
\mathcal{D}^{+} f(a):=\left\{w \in W: \exists_{h_{n} \backslash a} \frac{f\left(a+h_{n}\right)-f(a)}{h_{n}} \rightarrow w\right\} .
$$

It is easy to see that $\mathcal{D}^{+} f(a)$ is always a closed set.

Proposition 5.8. Let $X$ be a separable Banach space, $\operatorname{dim} X \geq 2$, and let $X=Y \oplus W$, where $\operatorname{dim} Y=1$ and $W$ is a closed linear subspace of $X$. Then there exists a Lipschitz function $h: Y \rightarrow W$ such that the "Lipschitz curve" $L:=\{y+h(y): y \in Y\}$ does not belong to the $\sigma$-ideal $\left(\widetilde{\mathcal{S}}_{+}(X)\right)_{I}$.

Proof. Choose $w_{0} \in W,\left\|w_{0}\right\|=1$ and a sequence $\left(w_{n}\right)_{n=1}^{\infty}$ of vectors from the ball $B\left(w_{0}, 1 / 2\right)$ which is dense in this ball. Further choose a sequence $a_{n} \searrow 0$ such that $a_{n+1} / a_{n} \rightarrow 0$. Let $p: \mathbb{R} \rightarrow W$ be the function with the following properties:

(i) $p(t)=w_{n}$ for $t \in\left[a_{2 n}, a_{2 n-1}\right], n \in \mathbb{N}$;

(ii) $p$ is affine on each interval $\left[a_{2 n+1}, a_{2 n}\right], n \in \mathbb{N}$;

(iii) $p(t)=0$ for $t \in(-\infty, 0]$ and $p(t)=w_{1}$ for $t \in\left[a_{1}, \infty\right]$. 
Clearly, $p$ is bounded. Put $P(x):=\int_{0}^{x} p(t) d t$. It is easy to show that $\mathcal{D}^{+} P(0)=$ $\overline{B\left(w_{0}, 1 / 2\right)}$. Now choose a sequence $\left(t_{n}\right)_{n=1}^{\infty}$ dense in $\mathbb{R}$ and put $g(t):=$ $\sum_{k=1}^{\infty} 2^{-k} p\left(t-t_{k}\right)$ and $G(x):=\int_{0}^{x} g(t) d t$. Thus, $G$ is Lipschitz. For each $n \in \mathbb{N}$, put

$$
G_{n}(x):=\int_{0}^{x} 2^{-n} p\left(t-t_{n}\right) d t, \widetilde{G}_{n}(x):=\int_{0}^{x} \sum_{k \neq n} 2^{-k} p\left(t-t_{k}\right) d t .
$$

It is easy to see that $\widetilde{G}_{n}^{\prime}\left(t_{n}\right)$ exists,

$$
\left(\mathcal{D}^{+} G_{n}\left(t_{n}\right)\right)=2^{-n} \mathcal{D}^{+} P(0)=2^{-n} \overline{B\left(w_{0}, 1 / 2\right)}
$$

and consequently

$$
\operatorname{int}\left(\mathcal{D}^{+} G\left(t_{n}\right)\right) \neq \emptyset \text {. }
$$

Now choose $y_{0} \in Y, y_{0} \neq 0$, define the mapping $h: Y \rightarrow W$ by $h\left(t y_{0}\right):=$ $G(t)$, put $L:=\{y+h(y): y \in Y\}$ and $z_{n}:=t_{n} y_{0}+h\left(t_{n} y_{0}\right)$. Then $h$ is Lipschitz. It is easy to show that $(5.3) \operatorname{implies} \operatorname{int}\left(\operatorname{contg}\left(L, z_{n}\right)\right) \neq \emptyset$. Since $\left(z_{n}\right)_{n=1}^{\infty}$ is dense in $L$ it is sufficient to apply Proposition 5.6 (i) (with $M:=L$ and $\left.D:=\left\{z_{n}: n \in \mathbb{N}\right\}\right)$ to see $L \notin\left(\widetilde{\mathcal{S}}_{+}(X)\right)_{I}$.

\section{References}

[A] N. Aronszajn, Differentiability of Lipschitzian mappings between Banach spaces, Studia Math., 57 (1976), 147-190.

[BC] D. N. Bessis and F. H. Clarke, Partial subdifferentials, derivates and Rademacher's theorem, Trans. Amer. Math. Soc., 351 (1999), 2899 2926.

[BL] Y. Benyamini and J. Lindenstauss, Geometric Nonlinear Functional Analysis, Vol. 1, Colloquium publications (American Mathematical Society), 48, Providence, Rhode Island, 2000

[Br] A. M. Bruckner, Some remarks on extreme derivates, Canad. Math. Bull., 12 (1969), 385-388.

[DV] V. F. Demyanov and L. V. Vasilev, Nondifferentiable Optimization, Optimization Software, Inc., New York, 1985.

[EG] L. C. Evans and R. F. Gariepy, Measure Theory and Fine Properties of Functions, Studies in Adv. Math., CRC Press, Boca Raton, 1992. 
[Fa] M. Fabian, Differentiability via one-sided directional derivatives, Proc. Amer. Math. Soc., 82 (1981), 495-500.

[Fe] H. Federer, Geometric Measure Theory Springer-Verlag, Berlin, 1969.

[FP] M. Fabian, D. Preiss, On intermediate differentiability of Lipschitz functions on certain Banach spaces, Proc. Amer. Math. Soc., 113 (1991), 733-740.

[G] P. G. Georgiev, The smooth variational principle and generic differentiability, Bull. Austral. Math. Soc., 43 (1991), 169-175.

[FZ] M. Fabian, N. V. Zhivkov, A characterization of Asplund spaces with the help of local $\epsilon$-supports of Ekeland and Lebourg, C. R. Acad. Bulgare Sci. 38 (1985), 671-674.

[J] V. Jarník, Sur l'extension du domaine de définition des fonctions d'une variable, qui laisse intacte la dérivabilité de la fonction, Bulletin international de l'Académie des Sciences de Boheme (1923), 1-5.

[K] P. Kenderov, The quasi-differentiable functionals are almost everywhere differentiable, Math. and Education in Math., 2 (1974), 123126.

[LW] K. S. Lau, C. E. Weil, Differentiability via directional derivatives, Proc. Amer. Math., Soc. 70 (1978), 11-17.

[M] J. Mařík, Derivatives and closed sets, Acta Math. Hungar. 43 (1984), $25-29$.

[N] A. Nekvinda, Properties of derivatives of Lipschitz functions (in Czech), an unpublished student's work, Charles University Prague, 1982.

[NZ1] A. Nekvinda and L. Zajíček, A simple proof of the Rademacher theorem, Časopis Pěst. Mat., 113 (1988), 337-341.

[NZ2] A. Nekvinda and L. Zajíček, Extensions of real and vector functions of one variable which preserve differentiability, Real Analysis Exchange, submitted.

[PZ] D. Preiss and L. Zajíček, Directional derivatives of Lipschitz functions, Israel J. Math., 125 (2001), 1-27.

[Sa] S. Saks, Theory of the Integral Hafner P. C., New York, 1937. 
[Sn] E. J. Mc Shane,Extension of range of functions, Bull. Amer. Math. Soc., 76 (1970), 334-339.

[Sp] A. Shapiro, On concepts of directional differentiability, J. Optim. Theory Appl., 66 (1990), 477-487.

[Z1] L. Zajičcek, On the points of multivaluedness of metric projections in separable Banach spaces, Comment. Math. Univ. Carolinae,, 19 (1978), $513-523$.

[Z2] L. Zajíček, Differentiability of the distance function and points of multivaluedness of the metric projection in Banach space, Czechoslovak Math. J., 33 (108) (1983), 292-308.

[Z3] L. Zajíček, Fréchet differentiability, strict differentiability and subdifferentiability, Czechoslovak Math.J., 41 (116) (1991), 471-489.

[Z4] L. Zajíček, On differentiability properties of Lipschitz functions on a Banach space with a Lipschitz uniformly Gâteaux differentiable bump function, Comment. Math. Univ. Carolinae, 38 (1997), 329-336.

[Z5] L. Zajíček, A note on intermediate differentiability of Lipschitz functions, Comment. Math. Univ. Carolinae. 40 (1999), 795-799.

[Z6] L. Zajíček, Products of non- $\sigma$-porous sets and Foran systems, Atti Sem. Mat. Fis. Univ. Modena, XLIV (1996), 497-505.

[Z7] L. Zajiček, On the points of multiplicity of monotone operators, Comment. Math. Univ. Carolinae, 19 (1978), 179-189.

[Zh1] N. V. Zhivkov, Generic Gâteaux differentiability of locally Lipschitzian functions, Collection: Constructive function theory '81 (Varna, 1981), $590-594$.

[Zh2] N. V. Zhivkov, Generic Gâteaux differentiability of directionally differentiable mappings, Rev. Roumaine Math. Pures Appl., 32 (1987), 179-188. 